The Astrophysical Journal, 672:590-603, 2008 January 1

(C) 2008. The American Astronomical Society. All rights reserved. Printed in U.S.A.

\title{
THE B AND Be STAR POPULATION OF NGC 3766
}

\author{
M. VIRGINIA MCSWAin ${ }^{1,2,3}$ \\ Department of Astronomy, Yale University, P.O. Box 208101, New Haven, CT 06520-8101; mcswain@lehigh.edu
}

WENJin HuANG

Department of Astronomy, MC 105-24, California Institute of Technology, Pasadena, CA 91125; wenjin@astro.caltech.edu

Douglas R. Gies and ERIKa D. Grundstrom ${ }^{4}$

Center for High Angular Resolution Astronomy, Department of Physics and Astronomy, Georgia State University, P.O. Box 4106, Atlanta, GA 30302-4106; gies@chara.gsu.edu, erika@chara.gsu.edu

AND

RichaRd H. D. TOWNSEND

Bartol Research Institute, University of Delaware, Newark, DE 19716; rhdt@bartol.udel.edu Received 2007 July 18; accepted 2007 September 26

\begin{abstract}
We present multiple epochs of H $\alpha$ spectroscopy for 47 members of the open cluster NGC 3766 to investigate the long-term variability of its Be stars. Sixteen of the stars in this sample are Be stars, including one new discovery. Of these, we observe an unprecedented $11 \mathrm{Be}$ stars that undergo disk appearances and/or near disappearances in our H $\alpha$ spectra, making this the most variable population of Be stars known to date. NGC 3766 is therefore an excellent location to study the formation mechanism of Be star disks. From blue optical spectra of 38 cluster members and existing Strömgren photometry of the cluster, we also measure rotational velocities, effective temperatures, and polar surface gravities to investigate the physical and evolutionary factors that may contribute to the Be phenomenon. Our analysis also provides improvements to the reddening and distance of NGC 3766, and we find $E(B-V)=0.22 \pm 0.03$ and $\left(V-M_{V}\right)_{0}=11.6 \pm 0.2$, respectively. The Be stars are not associated with a particular stage of main-sequence evolution, but they are a population of rapidly rotating stars with a velocity distribution generally consistent with rotation at $70 \%-80 \%$ of the critical velocity, although systematic effects probably underestimate the true rotational velocities, so that the rotation is much closer to critical. Our measurements of the changing disk sizes are consistent with the idea that transitory, nonradial pulsations contribute to the formation of these highly variable disks.
\end{abstract}

Subject headings: open clusters and associations: individual (NGC 3766) — stars: emission-line, Be

\section{INTRODUCTION}

NGC 3766 is a rich, young open cluster in the Carina spiral arm that is well known for its high content of Be stars (Slettebak 1985), and many previous studies of this cluster have focused on the characteristics of these stars to identify their evolutionary status. The cluster has been the target of numerous photometric studies (Ahmed 1962; Yilmaz 1976; Shobbrook 1985, 1987; Moitinho et al. 1997; Piatti et al. 1998; Tadross 2001; McSwain $\&$ Gies 2005b). But despite these intensive investigations, the cluster's age and distance remain somewhat uncertain; measurements of its age range from 14.5 to $25 \mathrm{Myr}$ (WEBDA; ${ }^{5}$ Moitinho et al. 1997; Tadross 2001), and its distance is between 1.5 and $2.2 \mathrm{kpc}$. The reddening $E(B-V)$ is between 0.16 and 0.22 (see the discussion of Moitinho et al. 1997).

Spectroscopic investigations of NGC 3766 have targeted a limited sample of cluster members, focusing primarily on the

\footnotetext{
1 Visiting Astronomer, Cerro Tololo Inter-American Observatory (CTIO). CTIO National Optical Astronomy Observatory, which is operated by the Association of Universities for Research in Astronomy (AURA), Inc., under cooperative agreement with the National Science Foundation (NSF).

2 NSF Astronomy and Astrophysics Postdoctoral Fellow.

3 Current address: Department of Physics, Lehigh University, 16 Memorial Drive East, Bethlehem, PA 18015.

4 Current address: Physics and Astronomy Department, Vanderbilt University, 1807 Station B, Nashville, TN 37235.

5 The WEBDA database is maintained by E. Paunzen and is available online at http://www.univie.ac.at/webda/navigation.html. See also Vizier Online Catalog, VII/92A (G. Lynga 1987).
}

Be star and supergiant populations (Harris 1976; Mermilliod 1982, and references therein; Slettebak 1985; Levesque et al. 2005). Even the eclipsing double-lined spectroscopic binary BF Centauri (=HD 100915), a member of NGC 3766, has been largely neglected by modern spectroscopic observations (Clausen et al. 2007 and references therein). For most cluster members, no detailed information about their physical characteristics such as temperature, gravity, rotation, and metallicity are known.

In this work we present red and blue optical spectra for both normal B-type and Be stars in the cluster. Like many prior studies of NGC 3766, our primary goal is to investigate the Be star population; but unlike other works, we achieve a more complete understanding of this subset of B stars by comparing these emission-line objects to their nonemission counterparts. Therefore, we present measurements of the effective temperature, $T_{\text {eff }}$, surface gravity, $\log g$, and in most cases the projected rotational velocity, $V \sin i$, for 26 normal B stars and 16 Be stars in NGC 3766. We use these results to improve the known reddening and distance to the cluster. From multiple epochs of $\mathrm{H} \alpha$ spectroscopy, we also investigate the variability of the circumstellar disks and estimate the disk massloss/gain rates for $11 \mathrm{Be}$ stars. Finally, we use the observed disk masses and angular momenta to show that nonradial pulsations are a possible origin of the disks, which probably fill during short-lived bursts of mass flow from the stellar surface.

\section{OBSERVATIONS}

We obtained spectra of NGC 3766 during multiple observing runs in 2003 March, 2005 February, 2006 May, and 2007 
TABLE 1

JOURNAL OF SPECTROSCOPY

\begin{tabular}{|c|c|c|c|c|c|c|c|c|}
\hline UT Dates & $\begin{array}{l}\text { Range } \\
(\AA)\end{array}$ & $\begin{array}{l}\text { Resolving Power } \\
\qquad(\lambda / \Delta \lambda)\end{array}$ & $\begin{array}{l}\text { Number of } \\
\text { Spectra }\end{array}$ & Telescope and Spectrograph & $\begin{array}{l}\text { Slit Plate } \\
\qquad(\mu \mathrm{m})\end{array}$ & Grating & Filter & Detector \\
\hline 2003 Mar $21-22$. & $5490-6790$ & 1800 & 20 & CTIO $1.5 \mathrm{~m}+$ Cassegrain & ... & $47 / 1$ & GG495 & Loral $1 \mathrm{~K} \times 1 \mathrm{~K}$ \\
\hline 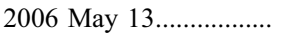 & $3790-4708$ & 3170 & 38 & CTIO Blanco 4 m + Hydra & $\ldots$ & KPGLD/2 & BG39 & SITe $4 \mathrm{~K} \times 2 \mathrm{~K}$ \\
\hline 2006 May $14-15 \ldots \ldots \ldots . .$. & $5125-8000$ & 1560 & 47 & CTIO Blanco 4 m + Hydra & $\ldots$ & KPGL3/1 & $\ldots$ & SITe $4 \mathrm{~K} \times 2 \mathrm{~K}$ \\
\hline 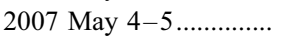 & $5125-8000$ & 2000 & 45 & CTIO Blanco 4 m + Hydra & 200 & KPGL3/1 & $\ldots$ & SITe $4 \mathrm{~K} \times 2 \mathrm{~K}$ \\
\hline 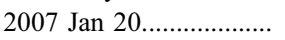 & $5650-6790$ & 1700 & 10 & CTIO $1.5 \mathrm{~m}+$ Cassegrain & ... & $47 / 1$ & GG495 & Loral $1 \mathrm{~K} \times 1 \mathrm{~K}$ \\
\hline 2007 Feb $2 .$. & $5650-6790$ & 1700 & 8 & CTIO $1.5 \mathrm{~m}+$ Cassegrain & $\ldots$ & $47 / 1$ & GG495 & Loral $1 \mathrm{~K} \times 1 \mathrm{~K}$ \\
\hline 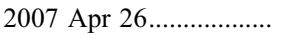 & $5650-6790$ & 1700 & 5 & CTIO $1.5 \mathrm{~m}+$ Cassegrain & $\ldots$ & $47 / 1$ & GG495 & Loral $1 \mathrm{~K} \times 1 \mathrm{~K}$ \\
\hline 2007 Jun 9 ......................... & $5650-6790$ & 1700 & 1 & CTIO $1.5 \mathrm{~m}$ + Cassegrain & $\ldots$ & $47 / 1$ & GG495 & Loral $1 \mathrm{~K} \times 1 \mathrm{~K}$ \\
\hline 2007 Jun 30 ...................... & $5650-6790$ & 1700 & 9 & CTIO $1.5 \mathrm{~m}+$ Cassegrain & $\ldots$ & $47 / 1$ & GG495 & Loral $1 \mathrm{~K} \times 1 \mathrm{~K}$ \\
\hline 2007 Jul 3 ......................... & $5650-6790$ & 1700 & 2 & CTIO $1.5 \mathrm{~m}+$ Cassegrain & $\ldots$ & $47 / 1$ & GG495 & Loral $1 \mathrm{~K} \times 1 \mathrm{~K}$ \\
\hline 2007 Jul $27-28 \ldots \ldots \ldots \ldots . .$. & $5650-6790$ & 1700 & 7 & CTIO $1.5 \mathrm{~m}+$ Cassegrain & $\ldots$ & $47 / 1$ & GG495 & Loral $1 \mathrm{~K} \times 1 \mathrm{~K}$ \\
\hline
\end{tabular}

January-July using the CTIO Blanco $4 \mathrm{~m}$ telescope with the Hydra multifiber spectrograph and the CTIO $1.5 \mathrm{~m}$ telescope with the Cassegrain spectrograph, operated by the SMARTS Consortium. The details of all runs are summarized in Table 1. Most of the runs targeted the $\mathrm{H} \alpha$ emission-line profile with low spectral resolution to characterize the Be stars' emission; however, during one run we observed the blue optical region with higher resolving power to observe numerous other $\mathrm{H} \mathrm{Balmer}$ and $\mathrm{He} \mathrm{I}$ line profiles and measure the physical parameters of the cluster members.

We selected the targets for each run by giving highest priority to the known Be stars in this cluster (save WEBDA star 232, which was saturated in our photometric study). We then selected other B-type stars in the cluster by ranking them according to their $y-\mathrm{H} \alpha$ color to preferentially select any weak emission stars that were not detected in our photometry (McSwain \& Gies 2005a, 2005b). All observations were performed by M. V. M. except the 2007 CTIO $1.5 \mathrm{~m}$ runs, which were taken in service mode by a SMARTS observer. For the Hydra observations, we generally began by taking short exposures and then parking the fibers used for the brightest stars to avoid saturation in the longer exposures. Not all of the known Be stars could be observed in one fiber configuration, so we took three to four exposures each of two configurations to observe all of the targets. Therefore, up to eight exposures of each star were obtained with the Hydra runs. We also observed a HeNeAr comparison lamp source just before and after the set of cluster observations for wavelength calibrations. For the CTIO $1.5 \mathrm{~m}$ observations, we alternated each stellar observation with a Ne comparison lamp spectrum.

The CTIO $1.5 \mathrm{~m}$ spectra were reduced and rectified to a unit continuum using standard routines for slit spectra in IRAF. ${ }^{6}$ All of the Hydra spectra were zero-corrected using standard routines in IRAF, and they were flat-fielded, wavelength-calibrated, and sky-subtracted in IRAF using the dohydra routine. In comparing the slit spectra and fiber spectra for many of the same objects in our data set, we find no evidence of systematic differences in the background subtraction due to cluster nebulosity. For each Hydra spectral configuration, we transformed the observations to a common heliocentric wavelength grid and co-added them to achieve a good signal-to-noise ratio for each star.

The complete sample of stars presented in this work is listed in Table 2. Column (1) gives each star's identification number based on the assigned number in McSwain \& Gies (2005b); the

\footnotetext{
${ }^{6}$ IRAF is distributed by the National Optical Astronomy Observatory, which is operated by AURA, Inc., under cooperative agreement with the NSF.
}

corresponding WEBDA numbers are given in column (2), where available. We obtained $\mathrm{H} \alpha$ spectra for each of these stars during at least three epochs in most cases, and these are shown in Figures $1 a-1 c$.

\section{PHYSICAL PARAMETERS FROM SPECTRAL MODELS}

\section{1. $V \sin i$ Measurements}

We began our investigation of each star's physical parameters by generating a grid of synthetic, plane-parallel, local thermodynamic equilibrium (LTE) atmospheric models using the Kurucz ATLAS9 code (Kurucz 1994). We adopted solar abundances and a microturbulent velocity of $2 \mathrm{~km} \mathrm{~s}^{-1}$ for these stars, which corresponds to the mean microturbulence observed among late-type, main-sequence (MS) B stars (Lyubimkov et al. 2004). Each atmospheric model was then used to calculate a grid of model spectra using SYNSPEC (Lanz \& Hubeny 2003).

For the 38 stars with available blue spectra, we made a preliminary estimate of their effective temperature and gravity, $T_{\text {eff }}$ and $\log g$, respectively, by comparing the observed $\mathrm{H} \gamma, \mathrm{H} \delta, \mathrm{He}$ I $\lambda 4143$, and He I $\lambda 4471+\mathrm{Mg}$ II $\lambda 4481$ line profiles to our grid of Kurucz spectral models. To measure $V \sin i$, we compared the observed $\mathrm{He}$ I line profiles to the model profiles convolved with a limb-darkened, rotational broadening function and a Gaussian instrumental broadening function. We determined the best fit over a grid of values, spaced $2 \mathrm{~km} \mathrm{~s}^{-1}$ apart, minimizing the mean square of the deviations, $\mathrm{rms}^{2}$. The formal error, $\Delta V \sin i$, is the offset from the best-fit value that increases the rms ${ }^{2}$ by $2.7 \mathrm{rms}^{2} / N$, where $N$ is the number of wavelength points within the fit region. Our measured $V \sin i$ and $\Delta V \sin i$ are listed in columns (3)-(4) of Table 3.

Even the He I lines may contain some weak emission in Be stars, partially filling and narrowing their line profiles. Furthermore, a number of the Be stars show evidence of narrow "shell" line components (formed in the outer disk), and the presence of a shell component may make the profile appear too narrow in some cases. Therefore, we consider our $V \sin i$ measurements for the $\mathrm{Be}$ stars to be lower limits. However, we note that the He I lines do not exhibit obvious signs of emission among most of the Be stars, and these lines are much less susceptible to emission than the $\mathrm{H} \gamma$ or $\mathrm{H} \delta$ lines. For the case of star 154 , a shell star with strong emission and contamination present in the He I lines, we used the Mg II $\lambda 4481$ line to measure $V \sin i$.

There are few previous measurements in the literature of $V \sin i$ for members of NGC 3766, but we found that 10 stars in 
TABLE 2

$\mathrm{H} \alpha$ Equivalent Widths

\begin{tabular}{|c|c|c|c|c|c|c|c|c|c|}
\hline MG ID & $\begin{array}{c}\text { WEBDA ID } \\
\text { (1) }\end{array}$ & $\begin{array}{c}W_{\lambda}(\AA) \\
(2003 \mathrm{Mar}) \\
(2)\end{array}$ & $\begin{array}{c}W_{\lambda}(\AA) \\
(2005 \mathrm{Feb}) \\
(3)\end{array}$ & $\begin{array}{c}W_{\lambda}(\AA) \\
(2006 \text { May) } \\
(4)\end{array}$ & $\begin{array}{c}W_{\lambda}(\AA) \\
(2007 \mathrm{Jan} / \mathrm{Feb}) \\
(5)\end{array}$ & $\begin{array}{c}W_{\lambda}(\AA) \\
(2007 \mathrm{Apr}) \\
(6)\end{array}$ & $\begin{array}{c}W_{\lambda}(\AA) \\
(2007 \text { May) } \\
(7)\end{array}$ & $\begin{array}{c}W_{\lambda}(\AA ̊) \\
\text { (2007 June) } \\
(8)\end{array}$ & $\begin{array}{c}W_{\lambda}(\AA) \\
(2007 \mathrm{July}) \\
(9)\end{array}$ \\
\hline 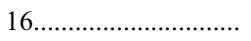 & 169 & $\ldots$ & 4.28 & 4.26 & $\ldots$ & $\ldots$ & 3.84 & $\ldots$ & $\ldots$ \\
\hline $23 \ldots \ldots \ldots \ldots$ & $\ldots$ & $\ldots$ & 9.22 & 9.37 & $\ldots$ & $\ldots$ & 7.59 & $\ldots$ & $\ldots$ \\
\hline 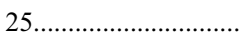 & 291 & 3.62 & 3.18 & 5.30 & $-1.63,-1.06$ & 0.42 & 1.04 & 1.42 & $\ldots$ \\
\hline $36 \ldots \ldots \ldots \ldots \ldots$ & 13 & $\ldots$ & 8.46 & 8.56 & $\ldots$ & $\ldots$ & 6.93 & $\ldots$ & $\ldots$ \\
\hline $41 \ldots \ldots \ldots \ldots \ldots \ldots \ldots \ldots \ldots$ & 130 & $\ldots$ & 4.88 & 5.28 & $\ldots$ & $\ldots$ & 4.41 & $\ldots$ & $\ldots$ \\
\hline $42 \ldots \ldots \ldots \ldots \ldots \ldots \ldots \ldots$ & 178 & $\ldots$ & 6.07 & 6.02 & $\ldots$ & $\ldots$ & 5.22 & $\ldots$ & $\ldots$ \\
\hline 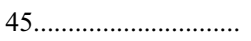 & 8 & 4.69 & 4.98 & 5.10 & $\ldots$ & $\ldots$ & 4.35 & $\ldots$ & $\ldots$ \\
\hline 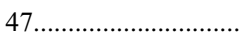 & 15 & -14.99 & -11.33 & -11.29 & -8.11 & $\ldots$ & -6.81 & -5.61 & -4.68 \\
\hline 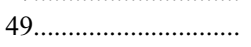 & 137 & 4.87 & 4.37 & 4.89 & $\ldots$ & $\ldots$ & 4.25 & $\ldots$ & $\ldots$ \\
\hline 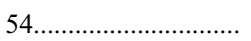 & 125 & $\ldots$ & 5.18 & 5.61 & $\ldots$ & $\ldots$ & 4.84 & $\ldots$ & $\ldots$ \\
\hline 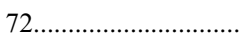 & 4 & 7.19 & 8.27 & 8.65 & 7.14 & $\ldots$ & 7.08 & $\ldots$ & 7.25 \\
\hline $73 \ldots \ldots \ldots \ldots \ldots \ldots \ldots$ & 26 & 3.65 & 0.42 & 3.28 & 1.27 & $\ldots$ & -0.35 & $\ldots$ & -0.92 \\
\hline 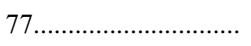 & 195 & 4.85 & 4.61 & 5.72 & $\ldots$ & $\ldots$ & 4.73 & $\ldots$ & $\ldots$ \\
\hline 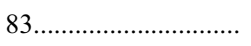 & 27 & 0.53 & 4.07 & 0.84 & 2.92 & 3.72 & 2.93 & $\ldots$ & $\ldots$ \\
\hline 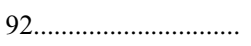 & 1 & 2.06 & 2.31 & 2.73 & 3.32 & $\ldots$ & 3.41 & 2.66 & $\ldots$ \\
\hline 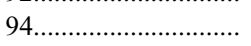 & 194 & $\ldots$ & 5.94 & 5.91 & $\ldots$ & $\ldots$ & 4.94 & $\ldots$ & $\ldots$ \\
\hline 96 & 45 & $\ldots$ & 6.39 & 6.87 & $\ldots$ & $\ldots$ & 6.26 & $\ldots$ & $\ldots$ \\
\hline 98................................. & 36 & 4.99 & 3.10 & 0.21 & 4.43 & 4.36 & 4.14 & $\ldots$ & 5.05 \\
\hline $101 \ldots \ldots$ & 34 & $\ldots$ & 7.40 & 7.44 & $\ldots$ & $\ldots$ & $\ldots$ & $\cdots$ & $\ldots$ \\
\hline 118 & $\ldots$ & $\ldots$ & 7.40 & 7.03 & $\ldots$ & $\ldots$ & 5.73 & $\ldots$ & $\ldots$ \\
\hline 119 & 81 & 4.76 & -5.48 & -3.19 & -6.53 & $\ldots$ & -8.67 & $\ldots$ & -12.83 \\
\hline $126 \ldots \ldots \ldots \ldots \ldots \ldots \ldots \ldots$ & $\ldots$ & $\ldots$ & 7.46 & 8.49 & $\ldots$ & $\ldots$ & 6.86 & $\ldots$ & $\ldots$ \\
\hline 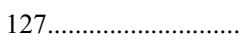 & 53 & -7.21 & -4.95 & -4.00 & -4.19 & $\ldots$ & -5.47 & -5.60 & -12.81 \\
\hline 170 & 94 & $\ldots$ & 4.81 & 5.26 & $\ldots$ & $\ldots$ & 4.17 & $\ldots$ & $\ldots$ \\
\hline 173 & 233 & $\cdots$ & 7.79 & 7.92 & $\cdots$ & $\cdots$ & 6.62 & $\cdots$ & $\cdots$ \\
\hline $175 \ldots \ldots \ldots \ldots \ldots \ldots \ldots \ldots$ & 218 & $\ldots$ & 6.67 & 6.74 & $\ldots$ & $\ldots$ & 6.01 & $\ldots$ & $\ldots$ \\
\hline 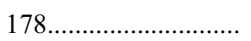 & 213 & $\ldots$ & 6.32 & 7.71 & $\ldots$ & $\ldots$ & 6.35 & $\ldots$ & $\ldots$ \\
\hline 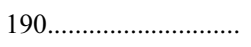 & 260 & $\ldots$ & 6.81 & 6.86 & $\ldots$ & $\ldots$ & $\ldots$ & $\ldots$ & $\ldots$ \\
\hline 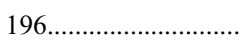 & 239 & 4.52 & 4.32 & 4.39 & $\ldots$ & $\ldots$ & 1.46 & $\ldots$ & $\ldots$ \\
\hline 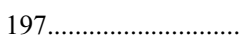 & 253 & $\ldots$ & 7.65 & 8.85 & 7.04 & $\ldots$ & 7.09 & $\ldots$ & 7.66 \\
\hline 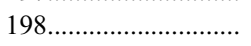 & 264 & -39.49 & -43.40 & -53.71 & -52.77 & $\ldots$ & -53.44 & -54.69 & $\ldots$ \\
\hline $200 \ldots \ldots \ldots \ldots \ldots \ldots \ldots$ & 240 & -8.84 & -6.66 & -4.62 & -4.88 & $\ldots$ & -5.19 & -5.76 & $\ldots$ \\
\hline
\end{tabular}

our sample were also measured by Slettebak (1985). Our $V \sin i$ measurements generally agree well, with the exception of star 154. Slettebak found $V \sin i=220 \mathrm{~km} \mathrm{~s}^{-1}$ for that star, nearly double our measured value. We emphasize that the exceptionally strong He and metal lines of this shell star amplify the difficulty of measuring its $V \sin i$.

\section{2. $T_{\mathrm{eff}}$ and $\log g$ Measurements of $\mathrm{B}$ stars}

For the B stars with $T_{\text {eff }}<15,000 \mathrm{~K}$, we used the "virtual-star" method of Huang \& Gies (2006) to improve our $T_{\text {eff }}$ and $\log g$ measurements. (Their virtual star is a spherically symmetric star with constant $T_{\text {eff }}$ and $\log g$ across the stellar surface.) They generated detailed $\mathrm{H} \gamma$ line profiles using line-blanketed, LTE Kurucz
ATLAS9 and SYNSPEC codes. Huang \& Gies show that the $\mathrm{H} \gamma$ line strength and equivalent width can be used as starting parameters in a line profile fit to obtain unique values of $T_{\text {eff }}, \log g$, and their corresponding errors. We used their procedure to measure these quantities from our observed $\mathrm{H} \gamma$ line profiles.

Among the hotter B-type stars, non-LTE effects alter the equivalent width of the $\mathrm{H} \gamma$ line, and thus the LTE Kurucz model line profiles systematically underestimate $T_{\text {eff }}$. Therefore, we used the new TLUSTY BSTAR2006 grid of metal line-blanketed, non-LTE, plane-parallel, hydrostatic model spectra (Lanz \& Hubeny 2007) to measure $T_{\text {eff }}$ and $\log g$ for those stars with $T_{\text {eff }}>15,000 \mathrm{~K}$. We used their models with solar metallicity and helium abundance and a microturbulent velocity of $2 \mathrm{~km} \mathrm{~s}^{-1}$. The grid includes $T_{\text {eff }}$ from 

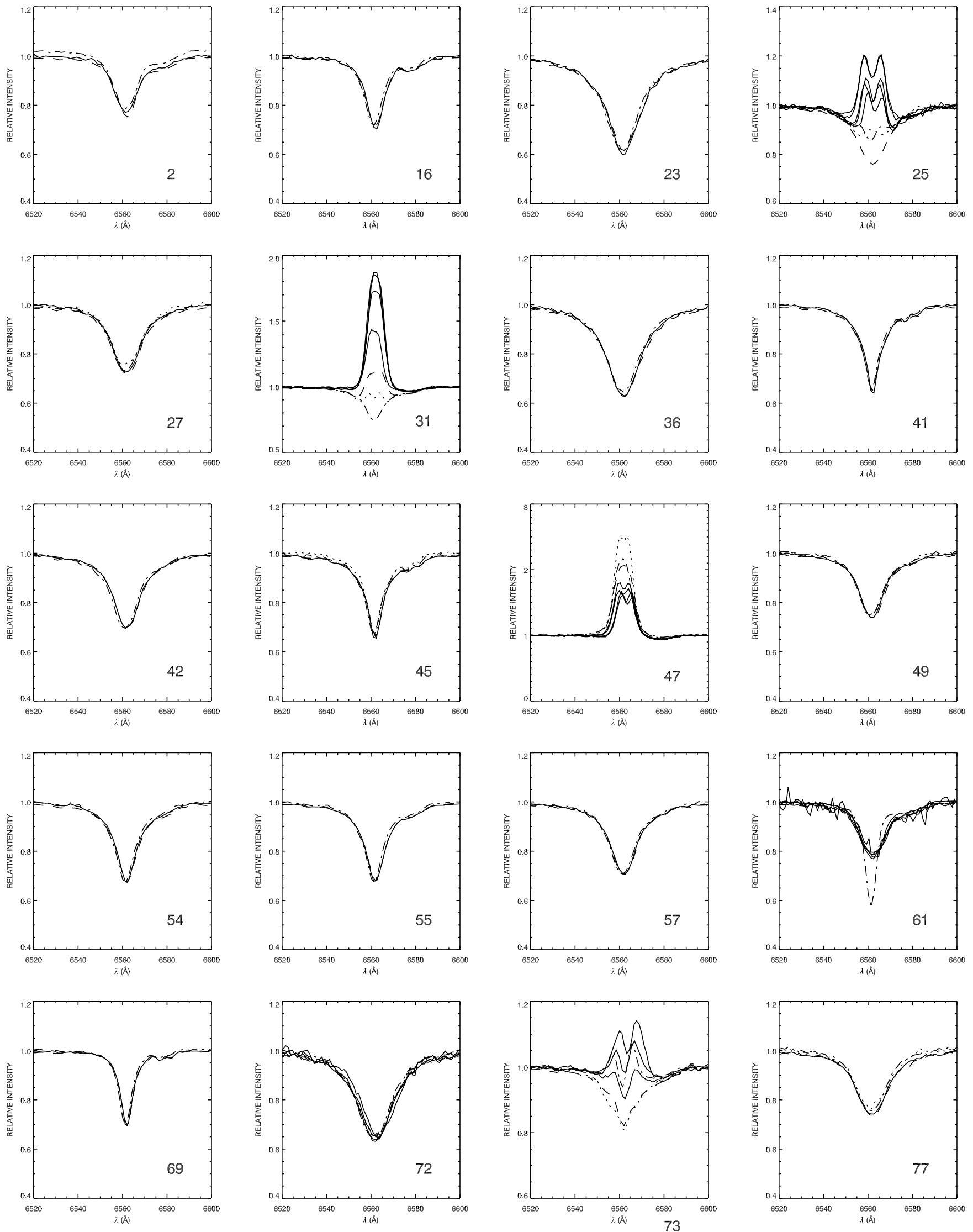

Fig. $1 a$

FIG. 1.- H $\alpha$ profiles, labeled by MG ID number. Spectra from 2003, where available, are shown with dotted lines, spectra from 2005 with dot-dashed lines, spectra from 2006 with dashed lines, and spectra from 2007 with solid lines. 

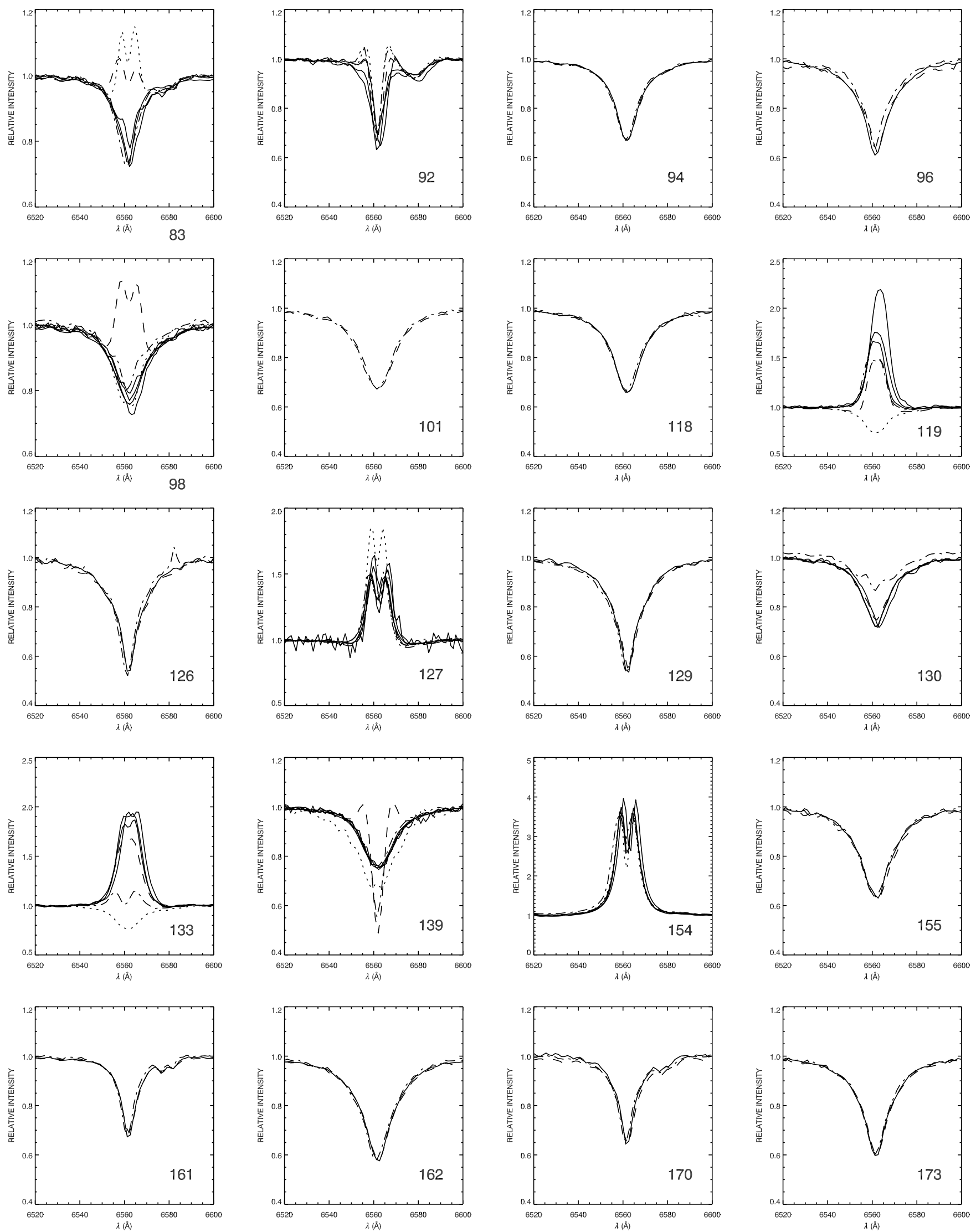

FIG. $1 b$ 

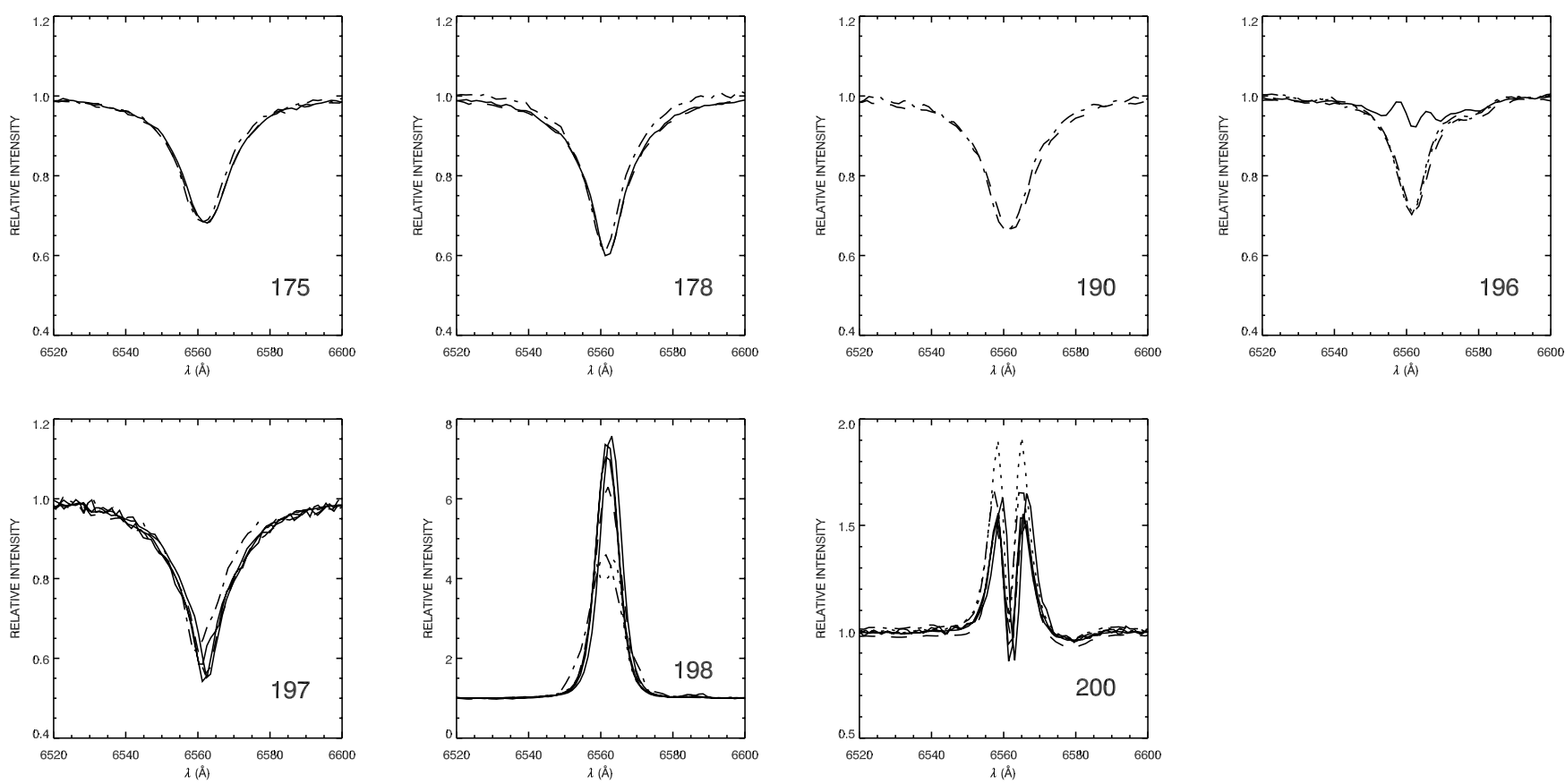

Fig. $1 c$

15,000 to $30,000 \mathrm{~K}$ in increments of $1000 \mathrm{~K}$ and $\log g$ from 1.75 to 4.75 in increments of 0.25 dex. For these hot stars we measured $T_{\text {eff }}$ and $\log g$ by comparing the $\mathrm{H} \gamma$ line profile to the rotationally and instrumentally broadened model spectral line profiles at each value in the grid, minimizing $\mathrm{rms}^{2}$ across the line region. We then refined our measurements to a higher precision using a linear interpolation between the available line profiles in the grid. Finally, we determined the errors, $\Delta T_{\text {eff }}$ and $\Delta \log g$, from the values that produce an $\mathrm{rms}^{2}$ no more than $2.7 \mathrm{rms}^{2} / N$ greater than the minimum $\mathrm{rms}^{2}$. Our measurements of $T_{\text {eff }}$ and $\log g$, with their corresponding errors, are listed in columns (5)-(8) of Table 3.

Many of the B stars in our sample are rapidly rotating, with $V \sin i>200 \mathrm{~km} \mathrm{~s}^{-1}$. Such a rapidly rotating star will experience strong centrifugal forces that distort the star into an oblate spheroidal shape, as recently found for the star Regulus (McAlister et al. 2005). The surface gravity at the equator can therefore be much lower than at the poles. For such a rapid rotator, our measured $T_{\text {eff }}$ and $\log g$ represent the average across the visible stellar hemisphere and are therefore biased toward lower values, causing the star to appear more evolved. Because the polar regions are not distorted, the surface gravity at the poles is a better indicator of the evolutionary state of the star. Huang \& Gies (2006) performed detailed spectroscopic modeling of such distorted rotating stars to determine a statistical correction factor for $\log g$, averaged over all possible values of $i$, for a variety of stellar models. We made a bilinear interpolation between their models to convert our measured $\log g$ to $\log g_{\text {polar }}$ for a more accurate comparison between slow and rapid rotators.

For each B star, we also measured its mass, $M_{\star}$, and radius, $R_{\star}$, by interpolating between the evolutionary tracks for nonrotating stars from Schaller et al. (1992). The errors $\Delta M_{\star}$ and $\Delta R_{\star}$ correspond to our measured $\Delta T_{\text {eff }}$ and $\Delta \log g$. Our results for $\log g_{\text {polar }}$, $M_{\star}, \Delta M_{\star}, R_{\star}$, and $\Delta R_{\star}$ are also listed in Table 3, columns (9)-(13).

\section{3. $T_{\mathrm{eff}}$ and $\log g$ Measurements of Be Stars}

Star 196 did not show any sign of Be emission until our most recent observations; hence, we included it among the normal $\mathrm{B}$ stars and measured $T_{\text {eff }}$ and $\log g$ from the $\mathrm{H} \gamma$ line in its 2006 blue spectrum. For other Be stars in our blue spectra, the above method to measure $T_{\text {eff }}$ and $\log g$ was not useful because the equivalent width of the $\mathrm{H} \gamma$ line may be decreased by emission in the line, even if the line profile does not exhibit obvious signs of emission.

Our first attempt to measure the Be stars' $T_{\text {eff }}$ and $\log g$ values relied on the He I $\lambda \lambda 4143,4388,4471$ lines. We fit these three lines using TLUSTY model spectra, following the same procedure described above for the $\mathrm{H} \gamma$ line. However, these $\mathrm{He}$ I line strengths are less sensitive to $T_{\text {eff }}$, and the line wings show only a very small dependence on $\log g$. Therefore, these line fits resulted in very large errors for both parameters in many cases. Furthermore, the Be disks contribute continuum flux that dilutes the apparent strength of the He I lines, so $\log g$ values measured from these lines do not always agree well.

To improve our $T_{\text {eff }}$ measurements for the Be stars, we turned to available Strömgren $m_{1}, c_{1}$, and $\beta$ indices for our targets (Shobbrook 1985, 1987; WEBDA). Several temperature relations for Strömgren indices are available in the literature (see Napiwotzki et al. 1993 and references therein), so we began by using our normal B-type stars with $T_{\text {eff }}>15,000 \mathrm{~K}$ as calibrators to determine the best relation for our data. We adopted the reddening value of $E(b-y)=0.15$ [corresponding to $E(B-V)=0.2$ ] for NGC 3766 (Shobbrook 1985; Moitinho et al. 1997). Eight stars in our sample $(16,42,49,54,57,161,170$, and 196) have available Strömgren indices as well as $T_{\text {eff }}$ measured from our $H \gamma$ line fits, and Napiwotzki et al. provide eight calibrators with well-known $T_{\text {eff }}>15,000 \mathrm{~K}$ (measured from their absolute integrated stellar flux) and available Strömgren data. For these 16 B stars, we found the best overall agreement using the temperature relation from Balona (1984) shown in Figure 2. However, we found that $T_{\text {Balona }}$ systematically underestimated $T_{\text {eff }}$, and a correction factor was necessary to improve their agreement. We performed a linear fit to the data and found the relationship

$$
T_{\text {eff, fit }}=1.052 T_{\text {Balona }}-359.636 \mathrm{~K}
$$


TABLE 3

Physical Parameters of Cluster Members

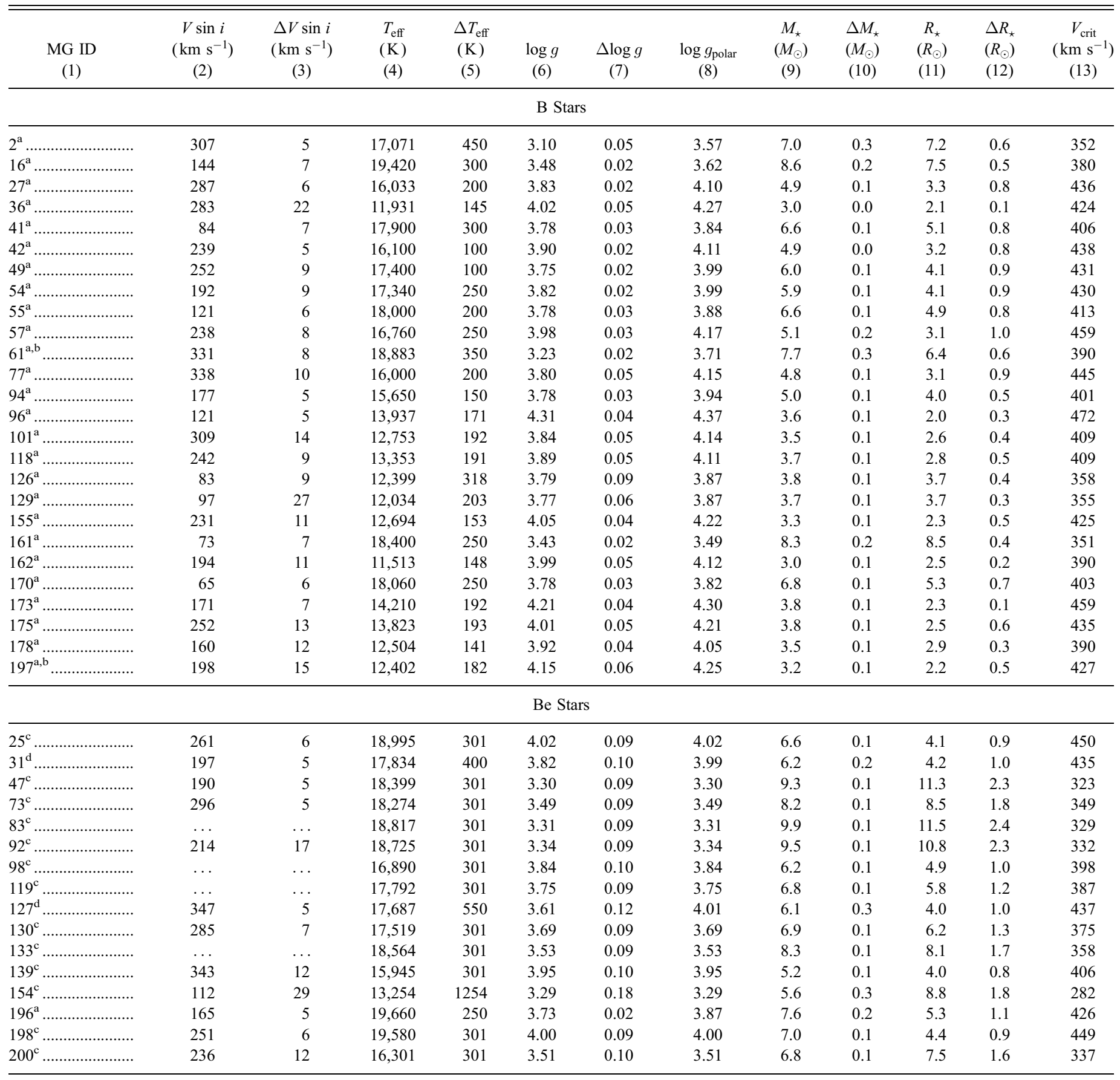

\footnotetext{
a $T_{\text {eff }}$ and $\log g$ measured from $\mathrm{H} \gamma$ line fit.

b Parameters are unreliable since the star is a suspected SB2.

c $T_{\text {eff }}$ and $\log g$ measured from Strömgren photometry.

${ }^{\mathrm{d}} T_{\text {eff }}$ and $\log g$ measured from $\mathrm{He}$ I line fits.
}

(excluding one outlying point from Napiwotzki et al.1993). The slope of this correction is virtually identical to the values found by both Gies \& Lambert (1992) and Cunha \& Lambert (1994). After applying this correction, we found a mean scatter of $264 \mathrm{~K}$ from our two independent measurements of $T_{\text {eff }}$ for the B stars in NGC 3766.

$T_{\text {Balona }}$ relies on the dereddened $c_{0}$ index as well as the narrowband $\beta$ magnitude, so determining an accurate temperature for the Be stars also requires confidence in $\beta$. However, the $\mathrm{Be}$ stars' emission makes the $\beta$ magnitude highly unreliable. There- fore, we used the B star calibrators from our sample (listed above) to investigate several $c_{0}-\beta$ relations in the literature (Crawford 1978 ; Balona \& Shobbrook 1984). The $c_{0}-\beta$ diagram is essentially a Hertzsprung-Russell (H-R) diagram that reveals temperature and evolutionary trends in a population. In Figure 3 we show that the values of $c_{0}$ and $\beta$ for these B-type calibrators generally agree with the relations for luminosity class III and V stars from Balona \& Shobbrook (1984). However, the calibration stars have $3.43<\log g<3.98\left(3.49<\log g_{\text {polar }}<4.17\right)$, since these hot stars are evolving along the MS, and neither $c_{0}-\beta$ relation can be 


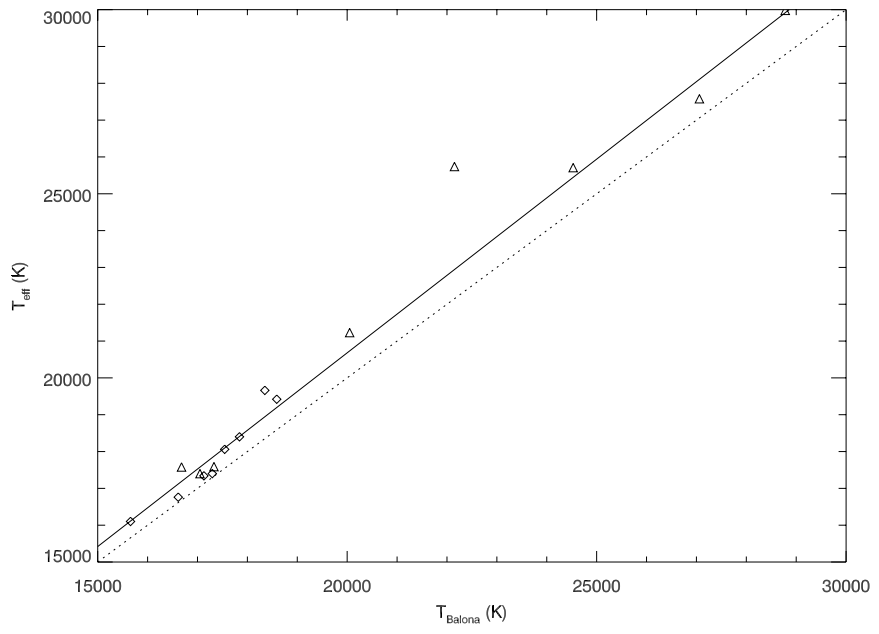

FIG. 2.- $T_{\text {eff }}$ measured for the B-type temperature calibration stars from this work (diamonds) and from Napiwotzki et al. (1993; triangles) compared to their calculated $T_{\text {Balona }}$ (Balona 1984). A linear fit to the two temperature scales (solid line) and the 1:1 agreement (dotted line) are also shown.

applied to the entire population. Therefore, we performed a linear fit to account for the range in evolution, and we found the relationship

$$
\beta_{\text {fit }}=0.417 c_{0}+2.545
$$

among the B stars in NGC 3766 with $T_{\text {eff }}>15,000 \mathrm{~K}$. The mean scatter between $\beta_{\text {fit }}$ and the measured $\beta$ is 0.010 , which implies an additional error of $144 \mathrm{~K}$ in $T_{\text {eff }}$, using the corrected $T_{\text {Balona }}$ relation above.

Shobbrook $(1985,1987)$ presented Strömgren photometry for nine Be stars that have accompanying blue spectra in this work (excluding 196, which we used as a calibrator) and four Be stars with only red spectra in this work. Thus, we measured $T_{\text {eff }}$ for all 13 of these Be stars using the adopted $\beta_{\text {fit }}$ and the corrected $T_{\text {Balona, }}$, as described above. We adopt a total error of $\left(264^{2}+144^{2}\right)^{0.5}=301 \mathrm{~K}$ for $T_{\text {eff }}$ measured with this method, and the results are listed in columns (5)-(6) of Table 3.

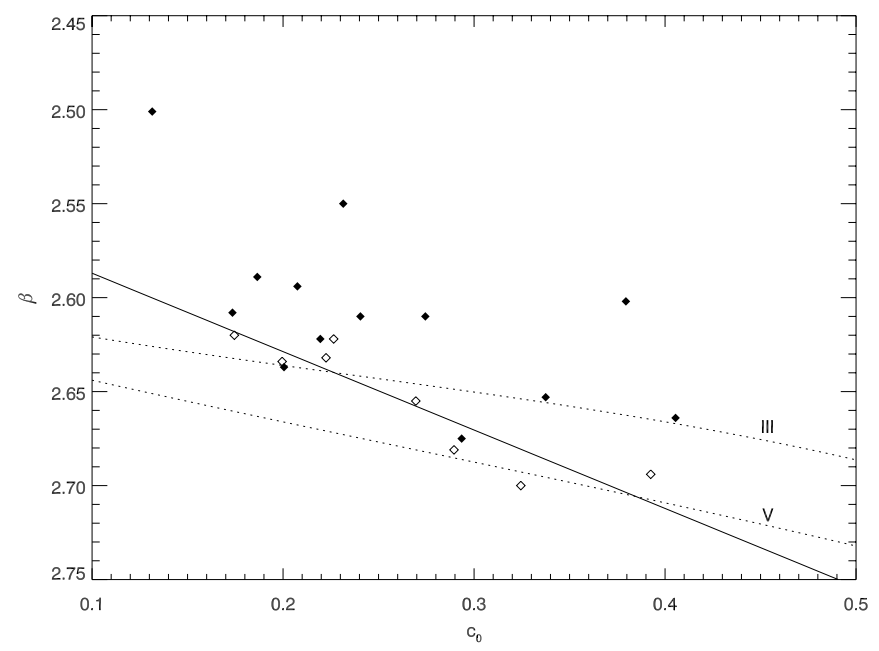

FIG. 3.- Strömgren $c_{0}$ index plotted against the $\beta$ magnitude for the B-type temperature calibration stars from this work (diamonds). Be stars are also plotted ( filled diamonds) to illustrate the contamination in $\beta$ due to their disk emission. We also show the $c_{0}-\beta$ relations for luminosity class V and III stars (Balona \& Shobbrook 1984; dotted lines) and our linear fit to this evolving population (solid line).

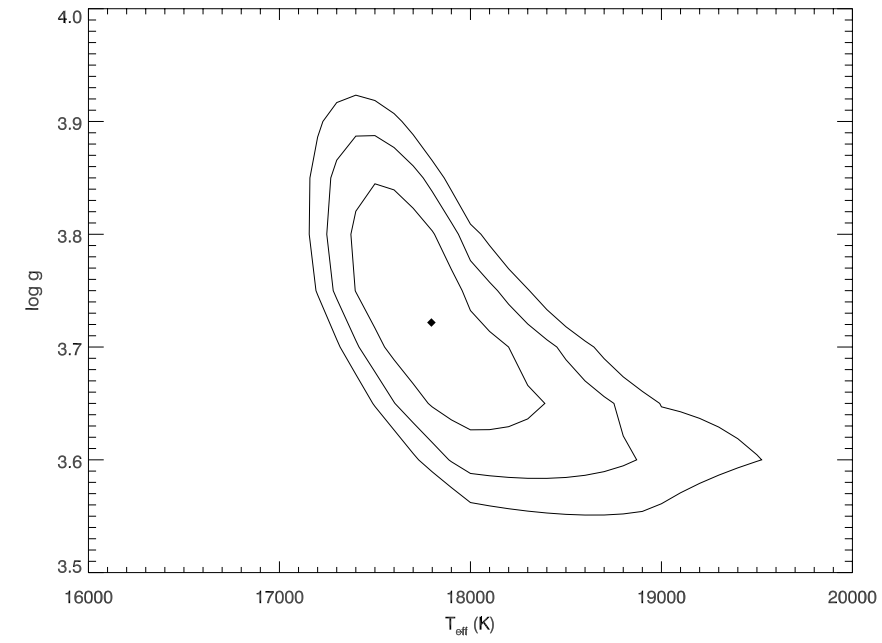

FIG. 4.-Contour plot of the 1,2 , and $3 \sigma$ errors in the He I $\lambda 4388$ line fit for star 31. We adopted the center of the $1 \sigma$ error region as the best-fit value, and the extent of this region indicates the sizes of the error bars for $T_{\text {eff }}$ and $\log g$.

Using this technique, only the "shell" Be star 154 results in $T_{\text {eff }}<15,000 \mathrm{~K}$. The comparisons between $T_{\text {Balona }}$ and our independently measured $T_{\text {eff }}$ are less reliable below this temperature threshold; hence, we used only B star calibrators with $T_{\text {eff }}>$ $15,000 \mathrm{~K}$. However, spectroscopy is even less likely to produce accurate measurements for 154 , since its spectrum is contaminated by $\mathrm{He}$ I emission and the metal lines are exceptionally strong due to the disk's edge-on orientation. Therefore, we include in Table 3 its $T_{\text {eff }}$ from the corrected relations of Balona (1984) but the errors are somewhat higher than for the other Be stars.

The values of $\log g$ are more strongly dependent on $\beta$, so we were reluctant to use our $\beta_{\text {fit }}$ with the Strömgren relation for $\log g$ given by Balona (1984). Instead, we used the calculated $T_{\text {eff }}$ to determine the Be stars' bolometric corrections (BCs), from Lanz $\&$ Hubeny (2007) at first assuming $\log g=4.0$. For the cooler star 154, we interpolated the BC from the values for MS stars given by Cox (2000). We calculated each stellar radius, $R_{\star}$, and luminosity, $L_{\star}$, using the measured $T_{\text {eff }}, \mathrm{BC}, V$ magnitude (Shobbrook 1985, 1987; WEBDA), distance modulus $\left(V-M_{V}\right)_{0}=11.73 \pm$ 0.33 (Moitinho et al. 1997), and $E(B-V)=0.2 \pm 0.1$ (Shobbrook 1985; Moitinho et al. 1997). We measured the stellar mass, $M_{\star}$, from the computed $T_{\text {eff }}$ and $L_{\star}$ by interpolating between the evolutionary tracks of Schaller et al. (1992). Finally, we obtained a preliminary value of $\log g$ from $M_{\star}$ and $R_{\star}$. Since the $\mathrm{BC}$ is weakly dependent on $\log g$, we improved the $\mathrm{BC}$ from the initial estimate and iterated to compute the final $\log g$. We adopt a formal error in $\log g$ computed from $\Delta T_{\text {eff }}$ and the quoted errors in $\left(V-M_{V}\right)_{0}$ and $E(B-V)$.

We note that the scatter between the calibrators' $\log g_{\text {polar }}$ and their Strömgren $\log g$ is identical to the formal error. Based on this good agreement, we do not perform any further correction to obtain $\log g_{\text {polar }}$ for the Be stars measured with this technique. However, we show below that the Be stars are more rapidly rotating than the normal B stars, which may make them appear slightly more evolved and artificially brightened. Thus, these values of $\log g_{\text {polar }}$ are lower limits. The final parameters for these Be stars are listed in Table 3.

Two Be stars in our sample of blue spectra have no available Strömgren data in the literature. By coincidence, these stars (31 and 127) have two of the best $T_{\text {eff }}$ and $\log g$ measurements from our preliminary He I line fits. Therefore, we adopt the mean measurements from the He I $\lambda \lambda 4143,4388,4471$ lines, with a formal error 


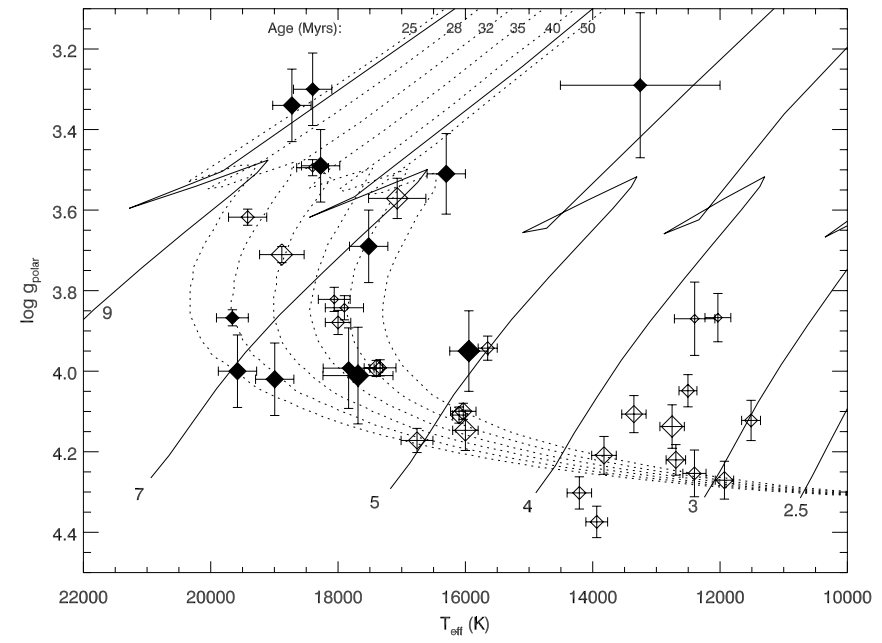

FIG. 5.- $T_{\text {eff }}$ and $\log g_{\text {polar }}$ are plotted with the evolutionary tracks of Schaller et al. (1992; solid lines) and isochrones of Lejeune \& Schaerer (2001; dotted lines). The ZAMS mass of each evolutionary track is labeled along the bottom, and the age of each isochrone is labeled along the top. Normal B-type stars are shown as open diamonds while Be stars are filled diamonds, and each symbol size is proportional to the star's $V \sin i$.

determined from the values which produce an $\mathrm{rms}^{2}$ no more than $2.7 \mathrm{rms}^{2} / N$ greater than the minimum $\mathrm{rms}^{2}$. A contour plot of the errors in $T_{\text {eff }}$ and $\log g$ for star 31 is shown in Figure 4, and the final values are listed in Table 3 . We determined their $\log g_{\text {polar }}$ using the same method as the B stars.

\subsection{Discussion of Physical Parameters}

The resulting values of $T_{\text {eff }}$ and $\log g_{\text {polar }}$ are plotted in Figure 5 with the corresponding evolutionary tracks for nonrotating stars from Schaller et al. (1992). The symbol sizes are proportional to $V \sin i$ to investigate the relation between rotation and the apparent evolutionary state of each star, but no trends are observed. Also in Figure 5, we plot the isochrones for 25-50 Myr populations from Lejeune \& Schaerer (2001). Our distribution of $T_{\text {eff }}$ and $\log g_{\text {polar }}$ are generally consistent with a population within this range, indicating an age slightly greater than previous estimates for NGC 3766 .

The masses of the rapidly rotating stars may be overestimated, since the evolutionary tracks do not account for rotation. We minimize this effect by using the corrected $\log g_{\text {polar }}$ for all stars, but slight mass differences may still be present, since rapid rotation is expected to alter the evolution (Heger \& Langer 2000; Meynet \& Maeder 2000). McAlister et al. (2005) found a small, 15\%, mass discrepancy for the rapid rotator Regulus when comparing its mass derived from nonrotating evolutionary tracks and its true mass from a detailed spectroscopic and interferometric analysis.

We find that the Be stars are generally among the hotter stars in our sample, consistent with our earlier findings from a photometric investigation of 48 open clusters that Be stars are preferentially found among the more luminous cluster members (McSwain \& Gies 2005b). There are no systematic differences between the $\log g_{\text {polar }}$ of the Be star and normal B star populations of the cluster, indicating that the Be stars in NGC 3766 are distributed across a range of $\log g_{\text {polar }}$ and are not associated with any particular stage of the MS evolution of B-type stars. This is also consistent with our earlier results (McSwain \& Gies 2005b) and with Zorec et al. (2005), who performed an evolutionary study of field Be stars. On the other hand, Levenhagen \& Leister (2006) found that field $\mathrm{Be}$ stars are preferentially found at later stages of the MS evolution. They did not perform any corrections for gravity darkening among these rapid rotators, so their Be stars may be found closer to the zero-age main sequence (ZAMS) than their results suggest.

\section{ROTATIONAL VELOCITIES}

Be stars are often described as a population of rapidly rotating B-type stars with a true rotational velocity $V_{\text {rot }}$ comparable to the critical velocity $V_{\text {crit. }}$ (Note that $V_{\text {rot }}$ should not be confused with $V$ in our measured $V \sin i$, which may be subject to systematic errors such as gravitational darkening or weak emission in the He I lines.) However, a recent study by Cranmer (2005) has cast some doubt on their fast rotation. He compared available $V \sin i$ measurements in the Yudin (2001) database of Oe, Be, and Ae stars to the predicted distribution of $V \sin i$ accounting for gravity darkening, limb darkening, and observational effects. He found that the Be stars of the Yudin database have intrinsic rotations between $40 \%-100 \%$ critical, with more early-type Be stars having significantly subcritical rotation. However, the Yudin database is a compilation of measurements from many different authors and instrumental setups, and therefore the available $V \sin i$ may contain significant systematic differences. Our measurements of $V \sin i$ rely on data of identical origin and measurements of the same spectral lines in nearly every case. Therefore, we have greatly reduced the systematic differences among our Be star measurements, and our measurements are also a reliable comparison of Be stars relative to normal B stars.

A rotationally distorted star has an equatorial radius $R_{e}=1.5 R_{p}$ in the Roche approximation; here $R_{p}$ is the star's polar radius. For simplicity, we assume $R_{p}$ is equal to the radius $R_{\star}$ derived from the position in Figure 5 and given in Table 3. The resulting critical velocity is

$$
V_{\text {crit }}=\sqrt{\frac{G M_{\star}}{R_{e}}}
$$

and is included in Table 3. A small mass discrepancy, as found for Regulus (McAlister et al. 2005), will not affect $V_{\text {crit }}$ significantly, since a $15 \%$ error in mass produces only a $4 \%$ error in $V_{\text {crit }}$. Here we investigate the rotational properties of several edge-on Be stars in our sample and compare the distribution of Be star velocities with the normal B stars to compare the two populations.

Two Be stars in our sample, 92 and 139, show $\mathrm{H} \alpha$ emission only in the line wings, with a deep absorption profile, suggesting the disks are observed nearly edge-on. This is probably a good assumption for star 139 , since it has $V / V_{\text {crit }} \geq 0.7$. However, star 92 may not be edge-on, since its $\mathrm{H} \alpha$ profiles between 1985 and 1990 (Balona et al. 1991) do not resemble the profiles we observe. Either the disk is precessing or other structural changes have occurred. A third star, 154, exhibits a shell spectrum with strong metal lines that also suggests an edge-on orientation.

If we assume that all of the Be stars have $V / V_{\text {crit }}=0.95$, then our measured $V \sin i$ indicate that stars 92, 139, and 154 have $i=34^{\circ}, 48^{\circ}$, and $15^{\circ}$, respectively. Assuming a slower $V / V_{\text {crit }}=$ 0.70 increases the derived values to $i=49^{\circ}, 90^{\circ}$, and $20^{\circ}$, respectively, but the low $i$ values for stars 92 and 154 are still inconsistent with the observed line profiles. However, if we fix $i=80^{\circ}$ for all three stars, we find that $V / V_{\text {crit }}=0.53$ for $92,0.71$ for 139 , and 0.24 for 154 . Townsend et al. (2004) show that measured $V \sin i$ may be too low due to gravitational darkening in highly distorted, rapidly rotating stars. But even accounting for a $20 \%-33 \%$ underestimate in $V \sin i$ for 92 and 154 , we do not find that these two Be stars are near critical rotation. 


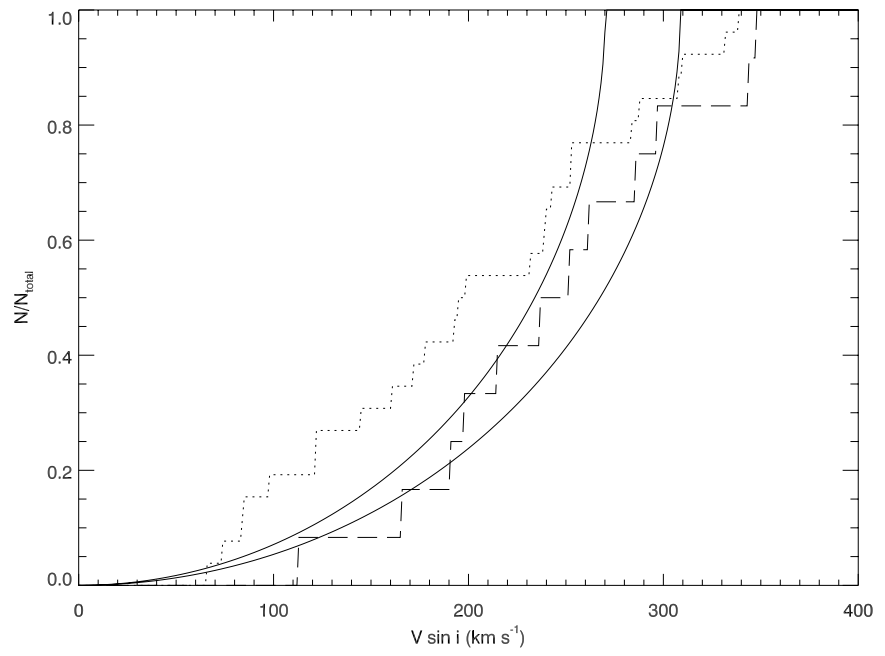

FIG. 6.-Cumulative distribution function of $V \sin i$ for the Be stars (dashed line) and the normal B-type stars (dotted line). Theoretical distributions of rapidly rotating stars with random orientation and $V=0.7 V_{\text {crit }}$ and $0.8 V_{\text {crit }}$ are also shown (solid lines; assumes the mean value for the Be stars, $V_{\text {crit }}=386 \mathrm{~km} \mathrm{~s}^{-1}$ ).

The distributions of $V \sin i$ for both the Be stars and normal B stars are plotted in Figure 6. Although this sample of 38 stars is small, this study is the first to measure $V \sin i$ for both groups using a consistent method and a uniform data set. Our measured $V \sin i$ for the Be stars may be only lower limits, but we clearly find that the Be stars of NGC 3766 are more rapidly rotating than the normal B star population. In Figure 6 we also include theoretical distributions of two uniform, rapidly rotating populations with $V=0.7 V_{\text {crit }}$ and $0.8 V_{\text {crit }}$, assuming $V_{\text {crit }}=386 \mathrm{~km} \mathrm{~s}^{-1}$ (the mean value among the Be stars, excluding star 154 due to its large measurement errors). The distribution of Be stars is consistent with $0.7 V_{\text {crit }}<V<0.8 V_{\text {crit }}$ in most cases. However, if each measured $V \sin i$ is underestimated by $20 \%-33 \%$ due to gravitational darkening, as claimed by Townsend et al. (2004), the distribution of Be star velocities is consistent with $V_{\text {rot }} \geq$ $0.84 V_{\text {crit. }}$. Stars 92 and 154 represent significant exceptions to this rule, as discussed above.

\section{OTHER RESULTS FROM SPECTRA}

We do not wish to broaden the scope of this paper to discuss the radial velocities and helium abundances observed in our spectra. However, we found several instances that suggest these topics are worth exploring in future studies of NGC 3766.

Figure $1 a$ includes the variable $\mathrm{H} \alpha$ profile of star 61 . The absorption line is deep and narrow in 2005, yet wide and shallow in 2006 and 2007. These line profile changes are not consistent with a Be star, and they probably indicate line blending in a double-lined spectroscopic binary (SB2). Likewise, star 197 may be an SB2. We observed double Mg II $\lambda 4481$ lines in 2006, and Figure $1 c$ shows a large wavelength shift in its $\mathrm{H} \alpha$ line profile between 2005 and 2006. There is possible line blending visible in the 2005 and $2007 \mathrm{H} \alpha$ profiles.

We measured the physical parameters of the B stars using spectral models with solar helium abundances, and most were consistent with a solar abundance. However, we noticed a large number that have abnormal He abundances; stars 41, 55, 94, 118, and 178 appear to be He strong, while 126, 129, 170, 173, and 197 appear He weak. Since all cluster members are expected to have the same He abundance, this may indicate the presence of widespread magnetic fields in the cluster, which can produce nonuniform distributions of He across the stellar photospheres (see the discussion in Huang \& Gies 2006). The He weak stars may also be binaries with cooler companions adding flux but diluting the appearance of the He r lines.

Finally, we used our spectra to reinvestigate the reddening and distance to NGC 3766. For the nonemission B stars with $\log g_{\text {polar }}>3.9$, we used the MS relation of Harmanec (1988) to assign a spectral type to each star based on its measured $T_{\text {eff }}$. We then used the MS relation of Wegner (1994) to find the stars' intrinsic colors, $(B-V)_{0}$. Observed $B-V$ colors were obtained from WEBDA. For the 17 B-type, MS stars in our sample, we found $E(B-V)=0.223 \pm 0.030$. We then used the B stars' $V$ magnitude (WEBDA), BC (Lanz \& Hubeny 2007; Malagnini et al. 1986), and our measured $R_{\star}$ to compute the distance modulus, $\left(V-M_{V}\right)_{0}$. The resulting $\left(V-M_{V}\right)_{0}=11.42 \pm 0.15$. To determine the mean $\left(V-M_{V}\right)_{0}$, we excluded star 45, with a somewhat lower $\left(V-M_{V}\right)_{0}$ of 10.3 , and thus probably a foreground star.

While the B stars of NGC 3766 are rotating more slowly than the Be stars, they have a mean $V \sin i / V_{\text {crit }}=0.5$. Assuming a random distribution of $i$, this corresponds to a mean $V / V_{\text {crit }}=$ 0.7 . Such rapidly rotating stars are probably rotationally distorted, with surface areas $\approx 1.2$ times larger than spherical star with $R_{\star}=R_{\text {polar }}$, causing them to appear overluminous. The distance modulus is then underestimated by a factor of $2.5 \log 1.2=$ 0.21 . With a realistic distribution of $V$ and $i$, this correction may not be appropriate for all stars, and the range in $\Delta\left(V-M_{V}\right)_{0}$ is $0.02-0.30$ for $0.28 \leq V / V_{\text {crit }} \leq 0.83$. We apply this correction to find a final $\left(V-M_{V}\right)_{0}=11.6 \pm 0.2$ for NGC 3766 , corresponding to a distance of $1.9-2.3 \mathrm{kpc}$. Our new values of $E(B-V)$ and $\left(V-M_{V}\right)_{0}$ are highly consistent with previous results for this cluster.

\section{Be STAR VARIABILITY}

While the long term $\mathrm{H} \alpha$ variability of Be stars has often been noted in the literature (e.g., Porter \& Rivinius 2003), few studies have attempted long-term monitoring of a large sample of $\mathrm{Be}$ stars to quantify their variability. Hubert \& Floquet (1998) investigated 273 bright Be stars with $V<7.5$ that were observed by Hipparcos between 1989 August and 1993 August. They found that early-type Be stars exhibit a very high degree of variability, while most late Be stars maintained a constant magnitude for the duration of the Hipparcos mission. They also identified $14 \mathrm{Be}$ stars with recurrent short-lived outbursts $(0.06 \leq \Delta \mathrm{Hp} \leq 0.3$ over timescales of 50-500 days) and 8 with long-lived outbursts ( $\Delta \mathrm{Hp} \geq 0.12$ over timescales of $>500$ days) in the sample. However, the Hipparcos magnitude, Hp, covers a broad wave band, ranging from 3400 to $8500 \AA$, and it traces large-scale variations in the continuum from scattered light associated with the disk. $\mathrm{H} \alpha$ spectroscopy is better suited to measuring variations over a larger dynamic range in the disks of Be stars of all spectral types.

Figures $1 a-1 c$ show the $\mathrm{H} \alpha$ line profile variations of all 47 stars in our spectroscopic sample. We were intrigued to discover a new Be star (130) in NGC 3766 based on our comparison of its $\mathrm{H} \alpha$ line profiles. In 2005 February the absorption line was broad and shallow, with some bumps that suggest a weak emission disk, nonradial pulsations, or simply noise. By 2006 May, however, the depth of the line had increased, while its width remained nearly the same, although a slight asymmetry in the line suggested that perhaps a very weak disk may still have been present. The line remained in absorption during our most recent 2007 observations. While an inverse relation between the line width and depth is associated with line blending in an SB2, the observed variations in star 130 cannot be attributed to such line 
blending. Instead, the three sets of observations indicate the presence of a weak circumstellar disk in 2005 that largely disappeared by 2006 .

In fact, we were startled to find that a total of $11 \mathrm{Be}$ stars in our sample exhibit significant changes in their disk state between 2003 and 2007. Stars 25, 31, 73, 83, 92, 98, 119, 130, 133, 139, and 196 each show $\mathrm{H} \alpha$ in absorption in at least one of the four epochs available, yet each star also experiences at least one epoch when the $\mathrm{H} \alpha$ line is partially or fully filled with emission. These observations suggest that the mechanism responsible for the disk formation is unstable over timescales of only a few years, consistent with the results of Hubert \& Floquet (1998). The disk growth of star 31 during 2007 reveals that disk formation can be very rapid, only requiring a few days or weeks, as observed in other Be stars (e.g., Grundstrom et al. 2007). However, the high fraction of Be stars that shows significant variability in a single cluster is unprecedented, and the cluster should be monitored with increased frequency to measure an accurate timescale of the disk state changes.

Five additional Be stars $(47,127,154,198$, and 200) show $\mathrm{H} \alpha$ emission in every observation, although the emission strength is usually variable. Such variations in $\mathrm{H} \alpha$ emission strength are typical among Be stars.

To quantify the observed changes in the Be star disks, we measured the equivalent width, $W_{\lambda}$, of the $\mathrm{H} \alpha$ line in each of our red spectra by normalizing each spectrum to a unit continuum and integrating over the line profile. Columns (3)-(6) of Table 2 give the measured values of $W_{\lambda}$ for each spectrum. The error in each measurement is about $10 \%$ due to noise in the continuum region.

With multiple epochs of $\mathrm{H} \alpha$ observations available for so many variable Be stars, it is worthwhile to estimate the changing size of the circumstellar disks. Grundstrom \& Gies (2006) describe a simple model to measure the ratio of the projected effective disk radius to the stellar radius, $R_{\text {disk }} / R_{\star}$, and the density at the base of the disk, $\rho_{0}$, using $W_{\lambda}$ and $T_{\text {eff }}$ as input parameters. We determined the disk inclination $i$ by assuming that each star is rotating with $V$ at $70 \%$ of $V_{\text {crit }}$ in most cases. We also assumed a disk truncation radius of $100 R_{\star}$, the nominal value unless a close binary companion is present. The disk temperature is assumed to be constant at $0.6 T_{\text {eff }}$.

To estimate the total masses of the disks, we used an axisymmetric, isothermal density distribution (Carciofi \& Bjorkman 2006),

$$
\rho(r, z)=\rho_{0}\left(\frac{R_{\star}}{r}\right)^{n} \exp \left\{-\frac{1}{2}\left[\frac{z}{H(r)}\right]^{2}\right\},
$$

and a radial density exponent, $n=3$, typical of other Be star disks (Gies et al. 2007). The scale height of the disk is

$$
H(r)=H_{0}\left(\frac{r}{R_{\star}}\right)^{\beta},
$$

where

$$
\begin{aligned}
& H_{0}=\frac{a}{V_{\text {crit }}} R_{\star}, \\
& a=\sqrt{\frac{k T}{\mu m_{H}}},
\end{aligned}
$$

and $\beta=1.5$ for an isothermal disk (Bjorkman \& Carciofi 2005; Carciofi \& Bjorkman 2006).
The resulting disk properties are listed in Table 4 for each Be star with an available $T_{\text {eff }}$ measurement. The time of each observation is listed in column (2), and the dates for the CTIO $4 \mathrm{~m}$ Hydra observations are less precise since these $W_{\mathrm{H} \alpha}$ values were measured from co-added spectra rather than individual spectra obtained with the CTIO $1.5 \mathrm{~m}$ telescope. Our determined values for $i, \rho_{0}, R_{\text {disk }} / R_{\star}$, and $M_{\text {disk }}$ are listed in columns (4)-(7).

Certainly, these disk measurements should be viewed with caution, since the true density profiles may be quite different from the assumed distribution. The disk density exponent may be different from our assumed value of $n=3$, and the resulting mass estimates may differ from our results by orders of magnitude. Contemporaneous optical and infrared spectra have also revealed evidence of density waves that alter the azimuthal disk structure (Wisniewski et al. 2007). Finally, the disks are likely not isothermal, as we assumed, and the true thermal structure may be very complex (Carciofi \& Bjorkman 2006). Therefore, the processes associated with the $\mathrm{H} \alpha$ emission profile are much more complex than assumed in the model of Grundstrom \& Gies (2006), although by using their simple model we obtain relative estimates of the variations in disk base density that are sensible, provided the disk density exponent is assumed constant in time.

\section{DISK FORMATION BY NONRADIAL PULSATIONS}

The estimates for $\rho_{0}$ and $M_{\text {disk }}$ given above are not sensitive to $i$ and offer a consistent method to measure changes in the size of the disks. Thus, we can estimate the disk growth rate (or dissipation rate), $\Delta M_{\text {disk }} / \Delta t$. Our generally sparse observations cannot determine whether $\Delta M_{\text {disk }} / \Delta t$ is constant or highly variable over timescales less than $1 \mathrm{yr}$; however there is an indication from the two closely spaced observations of stars 25 and 31 that the disk size can change rapidly. We provide our measured $\Delta M_{\text {disk }} / \Delta t$ between each pair of observations in Table 4 . The long-term buildup rates are comparable for every Be star in our sample, hinting that all of the disks might be formed by the same mechanism. Finally, the buildup and dissipation rates are also comparable in magnitude.

Many Be stars are known to exhibit nonradial pulsations (NRPs) with the mode $\ell=2, m= \pm 2$ (Rivinius et al. 2003), and such pulsations are commonly proposed as the source of kinetic energy required to inject material into the disk (Porter \& Rivinius 2003). The $m=+2$ pulsational mode is retrograde, and naively it might be expected to counteract the rotational velocity of the star and hinder disk formation. However, Townsend (2005) shows that retrograde mixed modes behave in a way that could contribute to Be disk formation. Although their phase velocity is retrograde, their group velocity is prograde. The density enhancements occur when the pulsational velocity perturbation is in the same direction as the rotation - a configuration favorable to mass ejection. Townsend also found that the mixed mode instability strip likely overlaps with the temperatures and spectral types of known $\mathrm{Be}$ stars if their rotation is nearly critical.

Observations of known $\beta$ Cephei pulsators and slowly pulsating $\mathrm{B}$ stars indicate that their pulsation modes have velocity amplitudes on the order of $10 \mathrm{~km} \mathrm{~s}^{-1}$ (combining the radial, azimuthal, and longitudinal components of the total velocity vector; De Ridder 2001). Owocki (2005) shows that the velocity needed for ejection into the disk is $\Delta V_{\text {orb }}=V_{\text {crit }}-V_{\text {rot }}$. For a weak atmospheric process such as NRPs, the atmospheric sound speed $a \sim 12 \mathrm{~km} \mathrm{~s}^{-1}$ must be comparable to $\Delta V_{\text {orb}}$. If we assume that the stars have $V_{\text {rot }}=0.95 V_{\text {crit }}$, then $\Delta V_{\text {orb }} \sim 20 \mathrm{~km} \mathrm{~s}^{-1}$, highly comparable to $a$ and the observed pulsation velocities in other NRP stars (De Ridder 2001). As we show above, this assumption of near critical rotation is reasonable for most of the Be stars in NGC 3766. 
TABLE 4

Estimated Sizes of Be Star Disks

\begin{tabular}{|c|c|c|c|c|c|c|c|}
\hline $\begin{array}{l}\text { MG ID } \\
\text { (1) }\end{array}$ & $\begin{array}{c}\text { HJD } \\
-2,450,000 \\
(2)\end{array}$ & $\begin{array}{c}\text { Assumed } \\
V / V_{\text {crit }} \\
\text { (3) }\end{array}$ & $\begin{array}{c}i \\
(\operatorname{deg}) \\
(4)\end{array}$ & $\begin{array}{c}\log \rho_{0} \\
\left(\mathrm{~g} \mathrm{~cm}^{-3}\right) \\
(5)\end{array}$ & $\begin{array}{l}R_{\text {disk }} / R_{\star} \\
\quad(6)\end{array}$ & $\begin{array}{c}M_{\text {disk }} \\
\left(10^{-11} M_{\odot}\right) \\
(7)\end{array}$ & $\begin{array}{c}\Delta M_{\mathrm{disk}} / \Delta t \\
\left(10^{-11} M_{\odot} \mathrm{yr}^{-1}\right) \\
(8)\end{array}$ \\
\hline \multirow[t]{8}{*}{$25 \ldots \ldots$} & 2720.825 & 0.7 & 64.9 & -12.2 & 2.3 & 41.9 & $\ldots$ \\
\hline & 3403.79 & $\ldots$ & $\ldots$ & -12.1 & 2.5 & 44.3 & 1.3 \\
\hline & 3870 & $\ldots$ & $\ldots$ & -12.3 & 1.6 & 30.6 & -10.7 \\
\hline & 4120.664 & $\ldots$ & $\ldots$ & -12.0 & 3.8 & 66.1 & 51.7 \\
\hline & 4129.743 & $\ldots$ & $\ldots$ & -12.0 & 3.7 & 63.7 & -92.7 \\
\hline & 4216.493 & $\ldots$ & $\ldots$ & -12.0 & 3.3 & 57.5 & -26.3 \\
\hline & 4225.66 & $\ldots$ & $\ldots$ & -12.1 & 3.1 & 54.7 & -110.0 \\
\hline & 4260.635 & $\ldots$ & $\ldots$ & -12.1 & 3.0 & 53.0 & -17.8 \\
\hline \multirow[t]{7}{*}{31} & 2720.744 & 0.7 & 40.5 & -12.1 & 2.5 & 19.8 & $\ldots$ \\
\hline & 3403.79 & $\ldots$ & $\ldots$ & -12.2 & 2.1 & 17.2 & -1.4 \\
\hline & 3870 & $\ldots$ & $\ldots$ & -12.0 & 3.0 & 24.6 & 5.8 \\
\hline & 4120.677 & $\ldots$ & $\ldots$ & -11.9 & 3.7 & 31.4 & 9.9 \\
\hline & 4129.771 & $\ldots$ & $\ldots$ & -11.9 & 4.1 & 35.0 & 142.4 \\
\hline & 4216.512 & $\ldots$ & $\ldots$ & -11.9 & 4.1 & 35.7 & 3.3 \\
\hline & 4225.66 & & $\ldots$ & -11.9 & 4.2 & 35.9 & 6.7 \\
\hline \multirow[t]{7}{*}{47} & 2719.557 & 0.7 & 40.8 & -11.8 & 4.5 & 72.3 & $\ldots$ \\
\hline & 3403.79 & $\ldots$ & $\ldots$ & -11.8 & 4.4 & 70.3 & -1.1 \\
\hline & 3870 & $\ldots$ & $\ldots$ & -11.8 & 4.4 & 70.2 & 0.0 \\
\hline & 4133.656 & $\ldots$ & $\ldots$ & -11.9 & 4.2 & 66.3 & -5.4 \\
\hline & 4225.66 & $\ldots$ & $\ldots$ & -11.9 & 4.1 & 64.1 & -8.8 \\
\hline & 4281.495 & $\ldots$ & $\ldots$ & -11.9 & 4.0 & 61.7 & -15.7 \\
\hline & 4309.466 & $\ldots$ & $\ldots$ & -11.9 & 3.9 & 59.5 & -28.5 \\
\hline \multirow{6}{*}{$73 \ldots \ldots$} & 2719.567 & 0.7 & 73.1 & -12.2 & 2.5 & 16.7 & $\ldots$ \\
\hline & 3403.79 & $\ldots$ & $\ldots$ & -12.0 & 3.5 & 23.0 & 3.4 \\
\hline & 3870 & $\ldots$ & $\ldots$ & -12.1 & 2.6 & 17.5 & -4.3 \\
\hline & 4120.711 & $\cdots$ & $\cdots$ & -12.1 & 3.3 & 21.5 & 5.8 \\
\hline & 4225.66 & $\ldots$ & $\ldots$ & -12.0 & 3.7 & 24.3 & 9.9 \\
\hline & 4308.554 & $\ldots$ & $\ldots$ & -12.0 & 3.9 & 25.3 & 4.1 \\
\hline \multirow[t]{6}{*}{92} & 2719.629 & 0.7 & 48.9 & -12.1 & 2.7 & 50.4 & $\ldots$ \\
\hline & 3403.79 & $\ldots$ & $\ldots$ & -12.1 & 2.6 & 49.1 & -0.7 \\
\hline & 3870 & $\ldots$ & $\ldots$ & -12.1 & 2.5 & 47.0 & -1.7 \\
\hline & 4133.668 & $\ldots$ & $\ldots$ & -12.1 & 2.3 & 43.9 & -4.3 \\
\hline & 4225.66 & $\ldots$ & $\ldots$ & -12.2 & 2.3 & 43.4 & -1.9 \\
\hline & 4281.510 & $\ldots$ & $\ldots$ & -12.1 & 2.5 & 47.3 & 25.9 \\
\hline \multirow[t]{7}{*}{$127 \ldots \ldots \ldots$} & 2719.759 & 0.8 & 80.1 & -11.9 & 5.6 & 30.7 & $\ldots$ \\
\hline & 3403.79 & $\ldots$ & $\ldots$ & -11.9 & 5.2 & 28.5 & -1.2 \\
\hline & 3870 & $\ldots$ & $\ldots$ & -11.9 & 5.0 & 27.4 & -0.9 \\
\hline & 4133.680 & $\ldots$ & $\ldots$ & -11.9 & 5.0 & 27.6 & 0.3 \\
\hline & 4225.66 & $\ldots$ & $\ldots$ & -11.9 & 5.3 & 29.1 & 5.8 \\
\hline & 4281.524 & $\ldots$ & $\ldots$ & -11.9 & 5.3 & 29.2 & 0.9 \\
\hline & 4309.495 & $\ldots$ & $\ldots$ & -11.8 & 6.1 & 33.8 & 59.9 \\
\hline \multirow[t]{5}{*}{$130 \ldots \ldots$} & 3403.79 & 0.7 & 63.5 & -12.1 & 3.0 & 11.9 & $\ldots$ \\
\hline & 3870 & $\ldots$ & $\ldots$ & -12.3 & 1.8 & 7.7 & -3.3 \\
\hline & 4120.750 & $\ldots$ & $\ldots$ & -12.2 & 2.2 & 9.0 & 1.9 \\
\hline & 4225.66 & $\ldots$ & $\ldots$ & -12.2 & 2.1 & 8.7 & -1.1 \\
\hline & 4281.540 & $\ldots$ & $\ldots$ & -12.2 & 2.1 & 8.8 & 0.6 \\
\hline \multirow[t]{7}{*}{139} & 2720.796 & 0.9 & 50.6 & $\ldots$ & 0.0 & 0.0 & $\ldots$ \\
\hline & 3403.79 & $\ldots$ & $\ldots$ & -12.3 & 1.8 & 1.7 & 0.9 \\
\hline & 3870 & $\ldots$ & $\ldots$ & -12.2 & 2.3 & 2.2 & 0.4 \\
\hline & 4120.770 & $\ldots$ & $\ldots$ & -12.2 & 2.2 & 2.1 & -0.1 \\
\hline & 4216.632 & $\ldots$ & $\ldots$ & -12.2 & 2.2 & 2.2 & 0.0 \\
\hline & 4225.66 & $\ldots$ & $\ldots$ & -12.2 & 2.3 & 2.2 & 0.0 \\
\hline & 4281.572 & $\ldots$ & $\ldots$ & -12.3 & 1.8 & 1.7 & -3.2 \\
\hline \multirow[t]{2}{*}{196} & 3870 & 0.7 & 33.5 & $\ldots$ & 0.0 & 0.0 & $\ldots$ \\
\hline & 4225.66 & $\ldots$ & $\ldots$ & -12.1 & 2.6 & 15.0 & 15.4 \\
\hline \multirow[t]{6}{*}{198} & 2720.658 & 0.7 & 69.9 & -11.9 & 5.0 & 253.4 & $\ldots$ \\
\hline & 3403.79 & $\ldots$ & $\ldots$ & -11.9 & 4.9 & 248.9 & -2.4 \\
\hline & 3870 & $\ldots$ & $\ldots$ & -11.9 & 4.7 & 238.7 & -8.0 \\
\hline & 4133.732 & $\ldots$ & $\ldots$ & -11.9 & 4.7 & 239.5 & 1.1 \\
\hline & 4225.66 & $\ldots$ & $\ldots$ & -11.9 & 4.7 & 238.9 & -2.4 \\
\hline & 4281.589 & $\ldots$ & $\ldots$ & -11.9 & 4.7 & 237.8 & -7.1 \\
\hline
\end{tabular}


TABLE 4-Continued

\begin{tabular}{|c|c|c|c|c|c|c|c|}
\hline $\begin{array}{c}\text { MG ID } \\
\text { (1) }\end{array}$ & $\begin{array}{c}\text { HJD } \\
-2,450,000 \\
(2)\end{array}$ & $\begin{array}{c}\text { Assumed } \\
V / V_{\text {crit }} \\
(3)\end{array}$ & $\begin{array}{c}i \\
(\operatorname{deg}) \\
(4)\end{array}$ & $\begin{array}{c}\log \rho_{0} \\
\left(\mathrm{~g} \mathrm{~cm}^{-3}\right) \\
(5)\end{array}$ & $\begin{array}{c}R_{\text {disk }} / R_{\star} \\
\quad(6)\end{array}$ & $\begin{array}{c}M_{\text {disk }} \\
\left(10^{-11} M_{\odot}\right) \\
(7)\end{array}$ & $\begin{array}{c}\Delta M_{\text {disk }} / \Delta t \\
\left(10^{-11} M_{\odot} \mathrm{yr}^{-1}\right) \\
(8)\end{array}$ \\
\hline \multirow[t]{6}{*}{200.} & 2720.637 & 0.7 & 46.2 & -11.8 & 4.8 & 9.9 & $\ldots$ \\
\hline & 3403.79 & $\ldots$ & $\ldots$ & -11.9 & 4.6 & 9.4 & -0.3 \\
\hline & 3870 & $\ldots$ & $\ldots$ & -11.9 & 4.3 & 8.7 & -0.5 \\
\hline & 4133.741 & $\ldots$ & $\ldots$ & -11.9 & 4.3 & 8.8 & 0.1 \\
\hline & 4225.66 & $\ldots$ & $\ldots$ & -11.9 & 4.4 & 8.9 & 0.4 \\
\hline & 4281.599 & $\ldots$ & $\ldots$ & -11.9 & 4.5 & 9.1 & 1.2 \\
\hline
\end{tabular}

To compare the NRP velocity with the outflow velocity required to fill the Be disks, we use the equation of mass continuity for an equatorial outflow with velocity $v_{r, \text { eq }}$,

$$
\sigma(r)=\frac{\Delta M_{\mathrm{disk}} / \Delta t}{2 \pi r v_{r, \mathrm{eq}}},
$$

where the surface density of the disk is

$$
\sigma(r)=\int_{-\infty}^{\infty} \rho(r, z) d z .
$$

Since we remove the vertical dependence of the disk density by using its surface density, this method effectively assumes that all of the material leaves the star through a cylindrical surface of arbitrary height. For a disk reaching down to the stellar surface, the mass must flow across this surface, so we use the surface density at the stellar surface, $\sigma\left(R_{\mathrm{eq}}\right)$, in the continuity relation. Since the $\ell=2, m= \pm 2$ modes have the greatest pulsational amplitude at the stellar equator, where the effective surface gravity is also a minimum, it is reasonable to expect the majority of the disk material to be ejected from the equator in the NRP model. The observed disk formation rates are generally $\Delta M_{\text {disk }} / \Delta t=10^{-12}$ to $10^{-11} M_{\odot} \mathrm{yr}^{-1}$, with $\rho_{0}=10^{-12} \mathrm{~g} \mathrm{~cm}^{-3}$ from Table 4 . This implies a very slow surface flow on average, $v_{r, \text { eq }} \sim .01-0.1 \mathrm{~km} \mathrm{~s}^{-1}$. However, a few observations reveal that the surface flow can be an order of magnitude faster, but this is still consistent with NRPs.

Likewise, we use our observed disk densities to demonstrate that NRPs are a capable source of angular momentum, $L$, for disks. The rotational inertia, $I$, of a thin disk shell is given by

$$
I(r)=2 \pi r^{3} \sigma(r) d r
$$

and the total angular momentum of the disk, extending from the stellar surface to $100 R_{\star}$, is

$$
L=\int_{R_{\mathrm{eq}}}^{100 R_{\star}} I(r) \omega(r) d r,
$$

where $\omega(r)=\left(G M_{\star}\right)^{0.5} r^{-1.5}$ is the angular velocity of a Keplerian disk. We find that $L \sim 10^{43}-10^{44} \mathrm{~g} \mathrm{~cm}^{2} \mathrm{~s}^{-1}$ for the observed disks, with $\Delta L / \Delta t \sim 10^{32}-10^{36} \mathrm{~g} \mathrm{~cm}^{2} \mathrm{~s}^{-2}$. Osaki (1986) shows that the angular momentum flux in the equatorial plane due to NRPs with $\ell=2, m= \pm 2$ is given by

$$
\frac{d L}{d t}=2 \pi R_{\mathrm{eq}}^{2} \sigma\left(R_{\mathrm{eq}}\right) A^{2} k \sin \delta .
$$

Here $A$ and $2 A k$ are the radial and azimuthal amplitudes of pulsation, respectively, and $\delta$ is the phase shift between the two ve- locity components. Thus, we find that a small azimuthal pulsation with $2 A k \sim 0.01-0.1 \mathrm{~km} \mathrm{~s}^{-1}$ can, in principle, provide enough angular momentum to eject material into the observed disks, and both the radial and azimuthal amplitudes are highly consistent with the observed pulsation amplitudes of other B stars with NRPs (De Ridder 2001).

Our results suggest that the NRP may be a transitory phenomenon for the Be stars, and the disks may fill substantially during short periods of surface activity. Evidence for changing pulsational modes among several stars in NGC 3766 has been observed by Balona et al. (1991), van Vuuren et al. (1988), and Balona \& Engelbrecht (1986). They found periodic light-curve variations in several Be stars in NGC 3766 (our stars 47, 92, 133, 154, and 200). Additional Be stars $(98,130,198)$ also exhibit light-curve variations that are possibly periodic. In several cases the shapes and possibly periods of the light curves change with timescales of only a few weeks. Balona et al. (1991) argue that this is due to magnetic fields, but the light curves are not qualitatively similar to variations observed in other magnetic stars, such as the prototype $\sigma$ Ori E (Oksala \& Townsend 2007). The changing light curves among NGC 3766 members are probably more consistent with changes in active pulsation modes over short timescales.

\section{CONCLUSIONS}

Our spectroscopic analysis of NGC 3766 has revealed that Be stars may be much more common than we originally thought. In our photometric study of NGC 3766 (McSwain \& Gies 2005b) we found up to $13 \mathrm{Be}$ stars (5 definite, 8 uncertain) out of an expected 191 B-type stars, not counting the 1 Be star that saturated our photometry. The new total of 16 Be stars is $23 \%$ greater. Among these 16 Be stars, 2-5 of them appear to have almost no disk at any given time, and an additional $2-4$ have extremely subtle emission in their $\mathrm{H} \alpha$ line profile that could easily be mistaken for other phenomena (such as NRPs manifesting themselves as bumps moving across the line or SB2 line blending). Therefore, $25 \%-50 \%$ of the Be stars may go undetected in a single spectroscopic observation, and photometric snapshots are even less likely to discern such weak emitters. We note four stars $(27,45,49$, and 77$)$ that were found to be possible or likely Be stars in the photometric study by Shobbrook $(1985,1987)$, but they never showed emission during our observations and thus remain unconfirmed. The existence of transitory, weak disks (especially stars 130 and 196) could mean that many more Be stars are waiting to be discovered.

For our total sample of 48 southern open clusters in our photometric survey, we found a low Be fraction of 2\%-7\% (McSwain $\&$ Gies 2005b). Considering the very weak disks that are observed in NGC 3766 and the exceptionally high variability among the cluster's Be population, the total fraction of Be stars could be much greater. We are currently performing a similar spectroscopic 
study of several other clusters from our survey, and we will address those results in a future paper.

While the Be stars of NGC 3766 are not distinguishable from normal B-type stars by their evolutionary states, they do form a population of rapidly rotating stars. With two exceptions, their measured velocities are consistent with a uniform population of rapid rotators having $V=0.7-0.8 V_{\text {crit }}$. Gravitational darkening and weak emission in the He I lines may mean that these velocities are underestimated by as much as $33 \%$ (Townsend et al. 2004), so the true $V_{\text {rot }}$ is probably at least $0.84 V_{\text {crit }}$. From the measured changes in the disks' masses and angular momenta, NRPs are adequate as a source for the mass flow into the equatorial plane. The pulsations may be a transitory phenomenon, however, and the variable nature of the Be stars probably reflects dramatic changes in the surface activity.
We thank the referee, Phil Massey, for providing comments that improved this work. Also, we are grateful to Giovanni Carraro, Mark Pinsonneault, and Swetlana Hubrig for helpful discussions that contributed to this work. We are grateful to Yale University and the SMARTS Consortium for providing observing time at the CTIO $1.5 \mathrm{~m}$ telescope. This research has made use of the WEBDA database, operated at the Institute for Astronomy of the University of Vienna. M. V. M. was supported by an NSF Astronomy and Astrophysics Postdoctoral Fellowship under award AST 04-01460. This work was also supported by the National Science Foundation under grant AST 06-06861 (D. R. G) and by NASA under grant LTSA/NNG05GC36G (R. H. D. T.).

\section{Facilities: CTIO:1.5m, CT10:4m}

Ahmed, F. 1962, Publ. R. Obs. Edinburgh, 3, 60

Balona, L. A. 1984, MNRAS, 211, 973

Balona, L. A., \& Engelbrecht, C. A. 1986, MNRAS, 219, 131

Balona, L. A., \& Shobbrook, R. R. 1984, MNRAS, 211, 375

Balona, L. A., Sterken, C., \& Manfroid, J. 1991, MNRAS, 252, 93

Bjorkman, J. E., \& Carciofi, A. C. 2005, in ASP Conf. Ser. 337, The Nature and Evolution of Disks Around Hot Stars, ed. R. Ignace \& K. Gayley (San Francisco: ASP), 75

Carciofi, A. C., \& Bjorkman, J. E. 2006, ApJ, 639, 1081

Clausen, J. V., Helt, B. E., Giménez, A., Vaz, L. P. R., García, J. M., Olsen, E. H., \& Southworth, J. 2007, A\&A, 461, 1065

Cox, A. N. 2000, Allen's Astrophysical Quantities (4th ed.; New York: Springer) Cranmer, S. R. 2005, ApJ, 634, 585

Crawford, D. L. 1978, AJ, 83, 48

Cunha, K., \& Lambert, D. L. 1994, ApJ, 426, 170

De Ridder, J. 2001, Ph.D. thesis, Katholieke Univ. Leuven

Gies, D. R., \& Lambert, D. L. 1992, ApJ, 387, 673

Gies, D. R., et al. 2007, ApJ, 654, 527

Grundstrom, E. D., \& Gies, D. R. 2006, ApJ, 651, L53

Grundstrom, E. D., et al. 2007, ApJ, 660, 1398

Harmanec, P. 1988, Bull. Astron. Inst. Czechoslovakia, 39, 329

Harris, G. L. H. 1976, ApJS, 30, 451

Heger, A., \& Langer, N. 2000, ApJ, 544, 1016

Huang, W., \& Gies, D. R. 2006, ApJ, 648, 591

Hubert, A. M., \& Floquet, M. 1998, A\&A, 335, 565

Kurucz, R. L. 1994, Kurucz CD-ROM 19, Solar Abundance Model Atmospheres for $0,1,2,4,8 \mathrm{~km} / \mathrm{s}$ (Cambridge: SAO)

Lanz, T., \& Hubeny, I. 2003, ApJS, 146, 417 2007, ApJS, 169, 83

Lejeune, T., \& Schaerer, D. 2001, A\&A, 366, 538

Levenhagen, R. S., \& Leister, N. V. 2006, MNRAS, 371, 252

Levesque, E. M., Massey, P., Olsen, K. A. G., Plez, B., Josselin, E., Maeder, A., \& Meynet, G. 2005, ApJ, 628, 973

\section{REFERENCES}

Lyubimkov, L. S., Rostopchin, S. I., \& Lambert, D. L. 2004, MNRAS, 351, 745 Malagnini, M. L., Morossi, C., Rossi, L., \& Kurucz, R. L. 1986, A\&A, 162, 140

McAlister, H. A., et al. 2005, ApJ, 628, 439

McSwain, M. V., \& Gies, D. R. 2005a, ApJ, 622, 1052 2005b, ApJS, 161, 118

Mermilliod, J.-C. 1982, A\&A, 109, 48

Meynet, G., \& Maeder, A. 2000, A\&A, 361, 101

Moitinho, A., Alfaro, E. J., Yun, J. L., \& Phelps, R. L. 1997, AJ, 113, 1359

Napiwotzki, R., Schoenberner, D., \& Wenske, V. 1993, A\&A, 268, 653

Oksala, M., \& Townsend, R. 2007, in ASP Conf. Ser. 361, Active OB Stars:

Laboratories for Stellar and Circumstellar Physics, ed. S. Stefl, S. Owocki, \&

A. Okazaki (San Francisco: ASP), 476

Osaki, Y. 1986, PASP, 98, 30

Owocki, S. 2005, in ASP Conf. Ser. 337, The Nature and Evolution of Disks Around Hot Stars, ed. R. Ignace \& K. Gayley (San Francisco: ASP), 101

Piatti, A. E., Claria, J. J., \& Bica, E. 1998, ApJS, 116, 263

Porter, J. M., \& Rivinius, T. 2003, PASP, 115, 1153

Rivinius, T., Baade, D., \& Stefl, S. 2003, A\&A, 411, 229

Schaller, G., Schaerer, D., Meynet, G., \& Maeder, A. 1992, A\&AS, 96, 269

Shobbrook, R. R. 1985, MNRAS, 212, 591 . 1987, MNRAS, 225, 999

Slettebak, A. 1985, ApJS, 59, 769

Tadross, A. L. 2001, NewA, 6, 293

Townsend, R. H. D. 2005, MNRAS, 364, 573

Townsend, R. H. D., Owocki, S. P., \& Howarth, I. D. 2004, MNRAS, 350, 189

van Vuuren, G. W., Balona, L. A., \& Marang, F. 1988, MNRAS, 234, 373

Wegner, W. 1994, MNRAS, 270, 229

Wisniewski, J. P., Kowalski, A. F., Bjorkman, K. S., Bjorkman, J. E., \& Carciofi, A. C. $2007, \mathrm{ApJ}, 656, \mathrm{~L} 21$

Yilmaz, F. 1976, A\&AS, 26, 1

Yudin, R. V. 2001, A\&A, 368, 912

Zorec, J., Frémat, Y., \& Cidale, L. 2005, A\&A, 441, 235 